\title{
1 Replay as structural inference in the hippocampal-entorhinal
}

\section{2 system}

3 Talfan Evans ${ }^{123, \#}$ and Neil Burgess ${ }^{12, \#}$

41 Institute of Cognitive Neuroscience, UCL

52 Institute of Neurology, UCL

63 CoMPLEX, UCL

$7 \quad$ \#correspondence

\section{Abstract}

9 Model-based decision making relies on the construction of an accurate representation of the

10 underlying state-space, and localization of one's current state within it. One way to localize is to

11 recognize the state with which incoming sensory observations have been previously associated.

12 Another is to update a previous state estimate given a known transition. In practice, both strategies are

13 subject to uncertainty and must be balanced with respect to their relative confidences; robust learning

14 requires aligning the predictions of both models over historic observations. Here, we propose a dualsystems account of the hippocampal-entorhinal system, where sensory prediction errors between these models during online exploration of state space initiate offline probabilistic inference. Offline inference computes a metric embedding on grid cells of an associative place graph encoded in the recurrent connections between place cells, achieved by message passing between cells representing non-local states. We provide testable explanations for coordinated place and grid cell 'replay' as efficient message passing, and for distortions, partial rescaling and direction-dependent offsets in grid

21 patterns as the confidence weighted balancing of model priors, and distortions to grid patterns as reflecting inhomogeneous sensory inputs across states. 


\section{Author Summary}

- Minimising prediction errors between transition and sensory input (observation) models predicts partial rescaling and direction-dependent offsets in grid cell firing patterns.

- Inhomogeneous sensory inputs predict distortions of grid firing patterns during online localisation, and local changes of grid scale during offline inference.

- Principled information propagation during offline inference predicts coordinated place and grid cell 'replay', where sequences propagate between structurally related features.

\section{Introduction}

Grid cells in the medial entorhinal cortex (mEC), whose firing fields form a periodic hexagonal lattice across the environment, are thought to support path integration ${ }^{1-3}$, whereas hippocampal place cells tend to have unimodal firing fields reflecting environmental cues such as boundaries ${ }^{4,5}$. Grid cell firing patterns are stable over time, suggesting corrective environmental inputs, possibly from place cells ${ }^{6}$, but rely more on self-motion than place cell firing patterns ${ }^{7}$ suggesting that environmental inputs are not fully corrective $e^{2,8,9}$. Given estimates of each input's uncertainty, Bayesian inference tells us how they should be optimally combined.

Although online learning (i.e. using only currently available sensory information) can converge under low PI and sensory noise, robust learning in the presence of noise requires minimizing the error between self-motion and environmental estimates of location across all state transitions ${ }^{10,11}$. Thus, historic observations must be stored and revisited offline (i.e. independently of current sensory inputs) to allow propagation of local environmental information to non-local but structurally connected regions of the cognitive map, e.g. as when adapting to a novel shortcut or barrier. This process can also be viewed as an embedding of sensory experience within a low-dimensional manifold (in this case, 2D space), as observed of place cells during sleep ${ }^{12}$.

Building on previous work ${ }^{11,13-15}$, we propose a dual-systems (online-offline) account of spatial inference in the hippocampal/entorhinal system, which we define as the process of identifying the 
configuration of both one's own location (current state) and the location of environmental landmarks in space (c.f. 'SLAM ${ }^{10}$ ). In familiar environments, online localization (identification of one's own position) is achieved by recursively combining self-motion and sensory inputs, which are mediated by learned transition and observation models, respectively. However, prediction errors between these models trigger offline inference over non-local states, facilitating fast learning of new or changed associative environmental structure, encoded online in place-place cell synaptic associations. We identify this offline inference with coordinated hippocampal/mEC 'replay'16-20.

Our framework also provides algorithmic- and implementation-level explanations for observed features of grid cell firing in response to manipulations ${ }^{7,9,21-24}$ or inhomogeneity $y^{25-29}$ of environmental sensory input. Overall, these phenomena can be understood in a probabilistic framework, where minimization of prediction errors between the transition and observation models are traded against prior model beliefs.

\section{Results}

\section{Probabilistic online localization with place and grid cells}

Grid cells exist in 'modules' of cells, whose firing patterns have the same spatial scale and orientation relative to the environment, but differ in their spatial offsets ${ }^{30}$. The spatial scale increases in discrete steps along the dorso-ventral axis, suggesting that, across modules, GCs support a hierarchical representation of space ${ }^{21,24,31-33}$. Here, we consider a single module of GCs, whose activity represent a probability distribution over a periodic, discretized region of space (visualised as a topographically arranged sheet of cells; Fig. 1A).

The self-location distribution is maintained over time by recursively integrating sensory and selfmotion inputs, accounting for their uncertainties (Fig. 1A). Firstly, the posterior distribution over agent location (grid module activity) from the previous time-step $\boldsymbol{G}$ is updated given noisy perceived movement $\widehat{\boldsymbol{u}}$ via the transition model $T$ (see Methods):

$$
G^{\prime}(\boldsymbol{x})=\int T\left(\boldsymbol{x}, \boldsymbol{x}^{\prime} \mid \widehat{\boldsymbol{u}}, \boldsymbol{\alpha}\right) \cdot G\left(\boldsymbol{x}^{\prime}\right) d \boldsymbol{x}^{\prime}
$$


where $\boldsymbol{x}$ is the 2D coordinate of the agent location in metric space (corresponding to a particular grid cell) and $\boldsymbol{x}^{\prime}$ the location at the previous time-step. Biophysically, $T$ would be represented by a population of direction dependent 'shifter' cells with asymmetric recurrent weights ${ }^{1}$ with a circulant structure $^{34}$ (Fig. S1C, see Methods) learned apriori (but see Refs. ${ }^{35-37}$ ). The rate of translation of activity on the grid sheet in response to movement $\widehat{\boldsymbol{u}}$ is controlled by the transition model gain $\boldsymbol{\alpha}=$ $\left[\alpha_{x}, 0 ; 0, \alpha_{y}\right]$, which might correspond to the strength of the associations to, or the speed dependence of, shifter or conjunctive cells ${ }^{31,38,39}$ (see Supplementary Methods).

The transition model estimate $G^{\prime}(\boldsymbol{x})$ is then refined by observations of environmental features, which map to metric space locations via observation model $H$ :

$$
G(\boldsymbol{x})=\frac{1}{K} H(\boldsymbol{P} \mid \boldsymbol{x}) \cdot G^{\prime}(\boldsymbol{x})
$$

where $\boldsymbol{P}$ is a vector of place cell firing rates, firing of place cell $i$ representing the likelihood of the presence of a specific sensory feature, or combination thereof. In our simulations, these have unique locations in physical space $\boldsymbol{\mu}_{i}$ and receptive field widths $\boldsymbol{\Sigma}_{P C}^{\mathrm{i}}$. Where the number of grid cells is large, the weights from place cell $i$ to the grid module define a distribution for that feature's estimated location in metric space (Fig. 1A). The weighted projection of place cell activity by these weights defines the observation model H. K provides inhibitory normalization (see Supplementary Methods).

Online learning modifies the observation model to reflect the current transition model location estimate (induces synaptic changes in the place-grid cell connection weights via a BCM rule; Fig. 2A; Methods). Online learning produces stable grid patterns (due to the circulant structure of $T$ ) for a range of levels of PI and sensory noise, but convergence fails in higher noise regimes (Fig. S2B). After a short period of initial learning, stable grid patterns emerge in the integrated estimate, despite the pure PI estimate being too noisy and the sensory associations too immature to drive stable patterns, if operating independently (Fig. 1B). 


\section{Offline inference: The hippocampus as a probabilistic graph}

Local, online learning is not robust in novel environments, because corrections to the estimated agent location (current grid cell activity), e.g. upon encountering familiar environmental features (place cell activity) associated to a different location on the grid module, also imply corrections to the encoding of feature observations along the preceding trajectory ${ }^{10}$. That is to say; local updates to the cognitive map also imply non-local, structurally associated changes. Formally, probabilistic spatial inference in this case requires finding the most likely configuration of metric space feature locations

$\left\{\boldsymbol{b}_{i}\right\}_{i=1: N_{P}}$ (each $\boldsymbol{b}_{i}$ is the 2D coordinate of feature $i$ in metric space, i.e. a place - grid cell association) and agent location $\boldsymbol{x}$ (the distribution over which is indicated by the grid cell firing rates) consistent with environmental sensory observations made along a given trajectory.

Theoretically, the configuration $\left\{\boldsymbol{b}_{i}\right\}_{i=1: N_{P}}$ can be recovered purely from the distances between pairs of environmental features ${ }^{10}$ (Fig. 1F, "square”, “ring”). Importantly, despite both feature locations being susceptible to large absolute errors (due to noisy PI), the errors will be correlated such that pairwise distance measurements will decrease in variance with observations ${ }^{10}$. This method predicts characteristic failure modes when the pairwise distance information is ambiguous or incomplete (e.g., Fig. 1F; "broken ring"). New distance observations might also cause dramatic changes to the inferred configuration (e.g. the discovery of a shortcut). If the current absolute estimates of feature node (i.e. place cell) location are stored in the place-grid cell synaptic weights, we propose that the relative distances between pairs of features are stored in the recurrent weights between place cells in hippocampal region CA3.

Consider a spring network, where the edge between environmental feature nodes $i$ and $j$ represents a noisy pairwise observation with length reflecting pairwise distance and stiffness reflecting certainty $^{13,40}$ (Fig. 1F, S6A). Minimizing the elastic energy in the spring mesh system corresponds to finding the maximum of the joint likelihood $L(\cdot)$, which is a function of the feature locations in metric space $\left\{\boldsymbol{b}_{i}\right\}_{i=1: N_{P}}$ and the internal gain parameter $\boldsymbol{\alpha}$, given pairwise distance measurements with 
121 Gaussian noise $\left(\delta_{i j}\right)$. This is equivalent to defining a probabilistic graphical model (see Methods) over the posterior:

$$
L\left(\boldsymbol{b}_{1}, \ldots, \boldsymbol{b}_{N_{p}}, \boldsymbol{\alpha}\right) \propto p\left(\boldsymbol{\alpha} \mid \boldsymbol{\alpha}_{0}\right) \prod_{(i, j) \in E} \psi\left(\boldsymbol{b}_{i}-\boldsymbol{b}_{j}, \boldsymbol{\alpha} \mid \delta_{i j}\right) \prod_{i=1: N_{p}} B_{i}\left(\boldsymbol{b}_{i}\right)
$$

124 where the current PC-GC weights $B$ ( $B_{i}$ is the probability distribution of the location of feature $i$ and $B_{i}\left(\boldsymbol{b}_{i}\right)$ is its value at metric/grid module location $\left.\boldsymbol{b}_{i}\right)$ act as priors on the feature node locations, the pairwise potential terms $\psi(\cdot)$ penalize the difference between associative pairwise distance measurements $\delta_{i j}$ made directly in environmental stimulus space, and the distance between their candidate locations in metric space $\boldsymbol{b}_{i}-\boldsymbol{b}_{j} . E$ is the set of connected PCs (see Methods). Distance in metric space is also a function of the transition model gain $(\boldsymbol{\alpha})$, which has a Gaussian prior $p\left(\boldsymbol{\alpha} \mid \boldsymbol{\alpha}_{0}\right)$ (a larger $|\boldsymbol{\alpha}|$ will decrease the metric space distance for all pairs; see Methods). Maximizing the likelihood (finding the state of minimum energy in the spring network) model over all feature node pairs minimizes the total prediction error between associative and metric generative models of the world ${ }^{15}$ (Fig. S7).

The associative distances can be straightforwardly learned during online exploration. Since Hebbian learning reflects coactivity, a trajectory exploring the environment uniformly results in synaptic strengths between place cells proportional to the spatial correlation between their receptive fields ${ }^{41}$. The Euclidean separation between their fields is then accessed via a simple transformation (see Methods; Fig. S1G). In this context, learning the PC-GC weights (modifying the observation model) during online localization corresponds to forming spatial priors over feature locations which anchor the structure,

140 which would otherwise be translation or rotation invariant (since measurements are relative), learned 141 during offline inference to constant locations on the grid-map. Taken together, our framework proposes a mapping onto anatomy of the joint agent-feature location distribution required for full probabilistic inference over environmental structure (Fig. S5; See overall algorithm in Table S1). 


\section{Partial grid pattern responses to environmental rescaling}

145 Uniform rescaling of an environment will introduce a mismatch between the estimates of location from the transition and observation models (i.e. a 'prediction error'). To minimize these prediction errors, the offline system can either modify the transition model gain to match the current environmental input (Fig. 2A, bottom), or modify the mapping from environmental inputs to metric space in the observation model (Fig. 2A, middle; see Methods). The degree to which either is modified should reflect their relative confidences, specified by a 'transition confidence score' $\left(\mathrm{T}_{\mathrm{C}}=\right.$ $\sigma_{\text {Obs }}^{2} / \sigma_{\alpha}^{2}$, the ratio of confidence in the transition vs observation models; see Methods). Similarly, a 'prior confidence score' $\mathrm{P}_{\mathrm{C}}$ specifies how much the system will tolerate persistent prediction errors; if $\mathrm{P}_{\mathrm{C}}$ is large, optimization may favour preserving prior configurations, as opposed to alignment of the current transition and observation models (see Methods).

We modelled experiments in which the physical environment ${ }^{21,42}$ and perceived velocity through a visual virtual environment ${ }^{7}$ were re-scaled, such that self-motion and sensory inputs conflicted. In both experiments, the rescaling of the grid patterns was partial, i.e. less than the magnitude of the physical or virtual manipulation, and less than those of place fields.

Both manipulations can be simulated by introducing a visual gain parameter $\alpha^{\text {Visual }}$ to the simulation of the environment (in both experiments it scales the amount of self-motion required to traverse the width of the perceived environment). Learned associative distances $(\boldsymbol{\delta})$ are also scaled by this parameter, reflecting its effect on the temporal overlap of place fields (see Methods Eq. 12). We simulated grid pattern rescaling responses over a range of transition confidence scores $\mathrm{T}_{\mathrm{C}}$. When confidence in the transition model is high $\left(\mathrm{T}_{\mathrm{C}} \rightarrow \infty\right)$, grid patterns in the real world are unchanged when plotted against physical movement (but are changed when plotted in visual VR coordinates; Fig. 2B, first column). The opposite is true when confidence in the observation model is high: grid patterns are unchanged relative to the apparent environment (Fig. 2B, last column). 
170 (Fig. 2B, middle column), matching the observed grid patterns in both experiments ${ }^{7,21}$ (and a similar 171 third experiment in on a virtual linear track ${ }^{43}$; Fig. 2C).

\section{Differential grid and place field responses to environmental reshaping}

How does the offline system respond to more complex environmental deformations? When one wall of a familiar rectangular environment is rotated inwards by $45^{\circ 22}$, place fields near the wall shifted almost fully while fields further away remained largely stationary, consistent with place fields preferentially reflecting local environmental inputs ${ }^{5}$. In contrast, grid fields shifted only partially near to the manipulated wall. Using the observed place field $\operatorname{shifts}^{23}$, we simulated the response of grid cells to the same manipulation (Fig. 3; see Supplementary Methods). Shifted place fields induce a misalignment between the associative distances and the distance between their encodings in metric space. The place field shifts are local and non-uniform, and so misalignment cannot be corrected by a global change to the transition gain $\boldsymbol{\alpha}$. Indeed, $\boldsymbol{\alpha}$ is not significantly modified during the optimization process, regardless of the $\mathrm{T}_{\mathrm{C}}$ value. Instead, alignment between the transition and observation models is maximized by modifying the observation model, i.e. updating the locations of the place fields on the grid module.

If there were no confidence in the prior observation model $\left(\mathrm{P}_{\mathrm{C}} \rightarrow 0\right)$, it would be modified offline to match the transition model, leaving the grid pattern unperturbed by the environmental change (Fig. 3B,C, top row). At the other extreme, favouring prior beliefs over recent observations (i.e. pairwise distances encoded during the manipulation trial) would result in an unchanged observation model, and grid field shifts that exactly mirror corresponding place field shifts (Fig. 3B,C, bottom row). In this regime, there would be permanent misalignment of the transition and observation models during online localization, producing noisy grid patterns, as when simulating a related experiment where grid distortions were observed in trapezoid environments ${ }^{44}$ (Fig. S2D). Setting $\mathrm{P}_{\mathrm{C}}$ to an intermediate value reproduces the experimentally observed partial shifting of grid fields (relative to the place fields ${ }^{23}$ ) when visualizing the structure encoded in the observation model (i.e. assuming low confidence in the transition model; Fig. 3B,C and D). 
In addition to partial changes to grid scale in response to environmental rescaling, enduring misalignments between observation and transition models can result from strong model priors, which prevent complete adaptation of the transition model gain. These cause the transition estimate to consistently precede that of the observation model, in the current direction of travel (on a 1D track, Fig. 2E) during VR visual gain decrease trials or physical expansion of the environment.

In all three cases, the integrated estimate of location (Eq. 2) in the online model converges to a fixed distance ahead of the observation model estimate (in the direction of travel; Fig. 2E inset and 3D), causing the grid pattern in the real world to dynamically shift opposite the direction of travel, as observed experimentally ${ }^{7,9}$. Our model suggests that the offsets should be partial (smaller than implied by a hard-reset at the boundary) and not specifically require a recent boundary encounter (cf. Keinath et al. $\left.{ }^{9}\right)$. Dynamic shifting in the model will reduce with experience of the novel or manipulated environment, as model misalignment reduces, as observed experimentally ${ }^{45}$.

\section{Online and offline perceptual warping in spatial representations}

210 With increasing experience of an environment, grid firing patterns exhibit both local scale changes ${ }^{26}$

211 and global shear-like distortions ${ }^{29}$, the latter associated with $7.5-8^{\circ}$ offsets of one of the grid axes ${ }^{29,44}$

212 to the walls of square environments. Both effects were present in our simulations and can be attributed

213 distinctly to the offline map-learning and online localization components of our theoretical

214 framework.

215 Firstly, we show that local changes to the grid scale ${ }^{26}$, which are positively correlated with

216 behavioural occupancy (animals spend more time in the middle of the environment), arise from the

217 offline process of map (PC-GC connections) learning. These mapping-induced distortions can be

218 further subdivided into two mechanisms, both of which induce local scale changes by biasing the 219 pairwise distances recovered from the Hebbian learned recurrent connections in CA3 (Fig. 1D,4; Fig. 220 S7). 
221 Firstly, relative behavioural under-sampling of the place fields near the boundaries of the environment

222 (using occupancy statistics from ${ }^{26}$; Fig. 4A, bottom) lead to weaker PC-PC connections, and

223 consequent overestimation of their pairwise distances, producing local scale changes (Fig. S7C).

224 Secondly, since Hebbian learned connection weights between place cells reflect the correlation in

225 their firing, and therefore their statistical discriminability ${ }^{46}$, two place cells with broad receptive fields

226 would develop a stronger connection than a pair with equal separation but narrower receptive fields

227 (stronger connections correspond to shorter distances on the grid module, producing grid patterns with

228 larger scales in the environment; Fig. 1D). Another recent study ${ }^{47}$ suggests that place fields are narrower near the edges of an environment, consistent with greater precision when driven by more proximal environmental features ${ }^{5}$ (Fig. 4A, top row). In our model, this produces weaker recurrent connections and a shrinking of the grid pattern at the edges of the environment following offline inference (Fig. 4D,E, top row).

Together, our results suggest that the cognitive 'distance' between two sensory features should be greater both when the absolute confidence in their spatial locations is greater (reflecting an increased statistical discriminability), or when those features are under-sampled relative to other features.

236 Although the action of both mechanisms are independent their effect is the same; both i) relative under-sampling of the transition between two adjacent states and ii) a reduced statistical discriminability between those states, both contribute to a weaker pairing of their representative place cells, resulting in greater separation between their encodings in metric space and a locally larger grid scale when 'read-out' in the firing pattern (a locally larger perception of distance).

241 In contrast, global shear-like distortions ${ }^{29}$ and associated $7.5-8^{\circ}$ offsets of one of the grid axes ${ }^{29,44}$ can

242 be interpreted as localization induced distortions during online exploration. In Stensola et al. ${ }^{29}$, rats

243 were introduced into the same corner of the box at the start of each trial; in Butler et al. ${ }^{25}$, shearing

244 developed following the introduction of reward ${ }^{25}$. In both experiments, shearing developed with increasing experience ${ }^{25,29}$. We hypothesized that these distortions reflect an increasing effect of non- 
246 uniform environmental inputs to the grid module, either reflecting their natural distribution ${ }^{25,29}$ or

247 inhomogeneous behavioural sampling of environmental locations ${ }^{26}$.

248 In our simulations, given a learned map, biasing the strength of sensory inputs at specific locations

249 (e.g. one/two corners) during online exploration reproduced several experimentally characterized

250 global distortions by causing a bias in the decoding of location (i.e. salient locations contribute a

251 larger 'vote'; Fig. S3; see Supplementary Methods).

252

\section{Probabilistic inference through HPC-mEC message passing}

To this point we discussed, from a functional perspective, how the brain might optimize its internal representations to reflect the uncertainty of sensory information. But how might the brain perform this optimization? In the above analyses of offline inference, we numerically computed the maximally likely feature locations on the grid module. However, the system must also track the uncertainty in these estimates, which would require updating the place-grid cell weights (including those with firing fields far from the agent location). An update of the full weight distributions is generally intractable when the state space is large.

Belief propagation ${ }^{48}$ is a technique for approximating this inference on graph structured data, and comprises two stages. First, a given feature node (i.e. a place cell) computes its location distribution (i.e. connections to the grid cells) $B_{i}\left(\boldsymbol{b}_{i}\right)$ by multiplying its prior $B_{i}^{(0)}\left(\boldsymbol{b}_{i}\right)$ with messages received from its connected neighbours (Fig. 5C; see Methods for details). A message $m_{i \rightarrow j}\left(\boldsymbol{b}_{j}\right)$ expresses neighbour node $i$ 's belief of node $j$ 's location, conditioned on its own distribution, and is dependent on the same pairwise potential terms $\psi(\cdot)$ in Eq. 3. The effect of a message is to favour distributions of nodes $i$ and $j$ which locate them at a radial distance equal to the associative distance $\delta_{i j}$; causing messages to be expressed as rings centred on the belief of the broadcasting node (Fig. 5C). Resolving a feature's unique location then depends on aggregating messages from multiple neighbours (Fig. 5C). Computations are distributed, and importantly only require information that is local to each neuron.

Each node in the graph iterates between updating its belief and broadcasting messages, converging when new messages cease to change the beliefs of their recipient nodes. As expected, the reduction in 
272

273

pairwise prediction error between associative distances and their corresponding distances in grid space (see Methods) over successive message iterations is accompanied by a sharpening of the distribution of each feature's location on the grid module (see Supplementary Methods; Fig. S1H).

\section{Offline inference triggered by prediction errors}

How might the online and offline systems interact? If the online system is sufficient to localize within pre-learned, simple or slowly changing environments, non-local reactivations of place cells would be unnecessary. However, more complex offline inference is required under more demanding circumstances, or in novel or changing environments. We hypothesize that offline or 'remote' inference is triggered by prediction errors between location estimates from the transition and observation models, respectively (Fig. 1G), defined in our model as the Kullback-Leibler divergence $\epsilon=\mathbb{K} \mathbb{L}\left(G^{\prime}, H\right)$

Prediction errors are large when the observation model prediction (weighted place cell input) is different and more sharply peaked than the transition model estimate (Fig. 1G; see Methods; prediction errors will not be generated in absence of incoming sensory information, as in darkness, when the observation model estimate is uncertain).

To illustrate our dual-systems (online+offline) hypothesis (Fig. S7), we simulated an agent navigating around a novel circular track (the loop closure task; Fig. 5). Completion of the first lap produces positive prediction errors between the sharply peaked input from feature inputs learned at the beginning of the trial, and the agent location estimate which is uncertain given the accumulation of PI noise (Fig. 5B).

Decrease in structural error (the difference between the place field separations and their encoded separations on the grid cell sheet) following online + offline inference was markedly larger than following online learning alone (Fig. 5E). The inferential power of this 'one-shot' learning process derives from consideration of the full covariance structure of the feature locations (captured by the CA3 connection weights between place cells), compared to the purely local learning occurring online. 
297 The system was subsequently able to navigate with dramatically reduced error (Fig. 5Aiv),

298 eliminating prediction errors on subsequent lap completions (Fig. 5B; Supplementary Video 1).

\section{Coordinated grid-place cell replay as structured information propagation}

300 The scheduling of updates in belief propagation is important because messages that do not change the beliefs of neighbours are redundant (Fig. 6A). We scheduled only the place cell whose belief had changed most to broadcast a new message on each cycle (Fig. 6A; see Methods). This max-update scheduling was more efficient than simple synchronous schemes, converging with fewer messages (Fig. 6C; see Ref. ${ }^{49}$ ).

The sequences of place cells broadcasting messages during offline inference in the loop-closure simulation have significant structure (6B). They tend to initially propagate backwards along the track from the animal's current position, resembling the characteristic reverse hippocampal replay following reward ${ }^{17}$ (Fig. 6B), but also occasionally hop to new locations where remote sequences are initiated $^{50}$ (Fig. 6B,F). These subsequent sequences showed an approximately equal distribution of forward/reverse sweeps (Fig. 6D; see Methods; Supplementary Video 1).

Thus, hippocampal 'replay' may reflect correction of local regions of the cognitive graph given new or 'surprising' information, as opposed to simple recapitulation of experience ${ }^{51}$. Sequences selectively affect place cells whose beliefs are structurally affected, and terminate when this is no longer the case, 'hopping' to remote regions. This leads to smooth sequences in un-converged graphs (novel environments) and more hoppy sequences with experience, where converged regions may be skipped

316 (Fig. 6F). These 'hops' marked the separation of 'replay' events into distinct sub-sequences (see

317 Methods). Multiple trajectories may also be played out in parallel (e.g. two trajectories alternating under max-scheduling; Fig. 6B, middle, grey shading).

How might belief propagation for offline inference be implemented in spikes fired by place and grid cells during replay? We propose a schematic model with a focus on function rather than biological 
of CA1). In the model, minimizing prediction errors between associative and metric generative models corresponds to synchronizing the propagation of activity through CA3 and $\mathrm{mEC}$, respectively (Fig. 7; see Supplementary Methods; Supplementary Video 2). A 'message' is initiated by a place cell spike, which propagates in CA3 via the Hebbian recurrent connections that encode place field separations. In parallel, the same spike initiates activity at the corresponding location on the grid cell module, which then propagates on the grid sheet as a traveling wave, using the same circuitry as path integration in the online model and propagating at the same speed as spikes in CA3 (see Methods). Hebbian-like learning strengthens connections from place cells to grid cell which simultaneously receive input in $\mathrm{CA} 3$ and $\mathrm{EC}$ respectively (Fig. 7A), approximating the algorithmic message-passing implementation (Fig. 7B, C). Firing of the broadcasting place cell is triggered by changes in its synaptic weights to the grid cell population, reflecting correction of the observation model in response to prediction error with the transition model (see Supplementary Methods).

\section{Discussion}

Building on previous work ${ }^{11,13,15}$, we argue that the mEC-HPC system performs spatial inference in two distinct regimes. Given a known 'cognitive map' (mapping sensory information to metric space), probabilistic integration allows optimal estimation of current location by online combination of uncertainty-weighted self-motion and environmental observations provided by transition and observation models respectively (Fig. 1). Where these estimates deviate strongly, prediction errors (Fig. 1G) trigger offline inference events (Fig. 5B), which propagate local environmental input to remote but structurally associated states, producing coordinated (often sequential) reactivations in place and grid cells (Fig. 6,7). The effect of offline inference is to produce a $2 \mathrm{D}$ embedding of the sensory information provided through the place cells, which may facilitate planning or generalization. Although not modelled here, back-projections from grid to place cells, reflecting the metric embedding of their place fields, might therefore also reduce uncertainty in place cells' firing, producing increased spatial stability in their fields, as observed to occur during sleep ${ }^{12}$.

Partial rescaling of grid patterns ${ }^{7,21}$ and differential shifting of grid and place fields ${ }^{23}$ in response to manipulations of environment sensory input can be understood as joint optimization of transition and 
observation models, balancing model priors with new observations. Where prediction errors persist, direction dependent grid pattern shifts may emerge as a result of probabilistic integration of these conflicting cues $^{7,9}$ (whereas boundary-dependent resetting ${ }^{9}$ produces larger shifts than experimentally observed and no rescaling; Fig. 2D, E).

We show that observed grid pattern distortions can be mechanistically linked to inhomogeneity in the sampling or neural representation of the environment ${ }^{25,26,29}$ (Figs. 4A, S3), which might be reflected in behaviour $^{52}$. Thus variation in the confidence, sampling or discriminability of sensory states will produce local changes in grid scale, inducing non-Euclidean structure in the metric representation of space (Fig. 1D, 4B). Our model also shows that distortions appear gradually with experience ${ }^{29}$, as the learned mapping from sensory features to metric space (the observation model) becomes more confident relative to the estimate of location from path integration (the transition model). Given initial learning, online localization errors (Fig. S3) should occur immediately following subsequent manipulations to the environmental sensory input, whereas offline changes may occur over longer timescales and correlate with replay of the manipulated states (Figs. 4,6; consistent with grid, but not place fields reorganizing significantly during sleep ${ }^{53}$ ). However, although large prediction errors will cause more easily detectable offline inference events, offline learning may occur continuously and not necessarily reactivate distinct previously experienced spatial trajectories ${ }^{51}$. We note that strong associative connectivity may also contribute to pattern completion, making the place cell representation robust to cue removal ${ }^{54}$.

Theoretical studies have demonstrated how the connectivity of the mEC metric space might emerge from a low-dimensional embedding of sensory stimuli ${ }^{55}$, predictive states ${ }^{35}$ or from unsupervised learning during navigational tasks ${ }^{56}$. A crucial difference in our model is that perceptually similar but physically separated compartments will be represented distinctly ${ }^{57}$, reflecting the vectorial translation between them in the transition model (i.e., not simply reflecting the topological state transition structure $\left.^{35}\right)$. Another recent model showed that grid cell like responses can emerge from learning the transition model that best predicts observed sensory stimuli ${ }^{36}$. We instead assume a fixed transition structure but with a variable linear gain, consistent with continuous attractor models ${ }^{31}$ where 
377

378

379

380

translation of activity on the grid cell sheet is driven by cells with velocity-dependent firing rates ${ }^{38,39}$. Indeed, a recent study showed that velocity dependence in $\mathrm{mEC}$ firing is tied to environmental manipulations ${ }^{39}$.

We propose that offline structural inference events correspond to coordinated $\mathrm{HPC} / \mathrm{mEC}$ replay ${ }^{16-19,58-}$ ${ }^{60}$, which can be viewed as synchronizing predictions from associative (CA3) and metric (mEC) generative models (Fig. S7). In this way, structural changes to an environment can be propagated to non-local regions of the metric embedding, in contrast to models in which these states need to be physically revisited ${ }^{13}$, consistent with the observation that replays do not necessarily repeat experienced trajectories ${ }^{51}$. Prediction errors between the two models may trigger replay events and corresponding sharp-wave ripples ${ }^{61,62}$. To our knowledge, this is the first functional model of coordinated place cell-grid cell replay ${ }^{18}$ (although cf. Ref. ${ }^{63}$ ), and provides an alternative to rewardbased theories $^{64,65}$ (we note that rewards may themselves represent salient sensory features, independent of their reward value).

Our model makes a number of experimentally testable predictions. Firstly, systematic manipulation of the discriminability of sensory cues distributed within an environment should produce predictable distortions to the grid pattern, observed with increasing experience of an environment. Secondly, replay should be more frequent after structural changes such as shortcuts, blockages or gain manipulations as in the experimental setup of Fig. 5. Thirdly, replay events triggered by specific unexpected sensory observations should become less frequent (Fig. 5B) and smooth (Fig. 6E, F) with continued experience, if the observations remain stable. Fourthly, multiple local replay events may occur in inter-leaved fashion (Fig. 6C, middle, grey shading). Fifthly, we predict the existence of travelling waves in grid cells (as a function of their spatial phase; see also ${ }^{66,67}$, Fig. 7). Lastly, initial messages propagating from the animal's current location may not cause subsequent messages in remote regions of the graph which are already sufficiently converged (messages will not cause changes in the beliefs of their recipients), although activity in $\mathrm{mEC}$ will continue to propagate. Thus grid cell replay could thus be detectable in the absence of simultaneous place cell replay ${ }^{19}$ (but place cell replay requires the grid cell transition model and so depends on $\mathrm{mEC}^{68}$ ). 
404 Our proposed structure learning framework can account for diverse phenomena observed in the HPC-

405 mEC system, and makes several novel, experimentally testable predictions.

406

\section{Methods}

\section{Online recursive Bayesian estimation}

The transition matrix $T$ defines the probability of transitioning from agent location $\boldsymbol{x}^{\prime}$ to location $\boldsymbol{x}$, and is a function of the perceived current velocity $\widehat{\boldsymbol{u}}$ and transition model gain $\boldsymbol{\alpha}=\left[\alpha_{x}, 0 ; 0, \alpha_{y}\right]$.

Since our metric space is periodic, $T$ accounts for cyclic transitions $\boldsymbol{c}_{m n}$, with Gaussian noise proportional to the perceived velocity $\widehat{\boldsymbol{u}} \sim \boldsymbol{u}+N\left(0, \operatorname{diag}(\boldsymbol{u}) \boldsymbol{\Sigma}_{\mathrm{PI}}\right)$ :

$$
T\left(\boldsymbol{x}, \boldsymbol{x}^{\prime} \mid \widehat{\boldsymbol{u}}, \boldsymbol{\alpha}\right)=\sum_{m n=-\infty}^{\infty} f\left(\boldsymbol{x}-\boldsymbol{x}^{\prime} \mid \widehat{\boldsymbol{u}}+\boldsymbol{c}_{m n}, \operatorname{diag}(\widehat{\boldsymbol{u}}) \boldsymbol{\alpha}^{-1} \boldsymbol{\Sigma}_{\mathrm{PI}}\right)
$$

where $f(\boldsymbol{x} \mid \boldsymbol{\mu}, \boldsymbol{\Sigma})$ is a multivariate Gaussian PDF, $\boldsymbol{c}_{m n}=2 \boldsymbol{\alpha}^{-1}\left(m \boldsymbol{v}_{1}+n \boldsymbol{v}_{2}\right)$ and $\boldsymbol{v}_{1}=$

$[\cos (\phi), \sin (\phi)]$ and $v_{2}=\left[\cos \left(\phi+\frac{\pi}{3}\right), \sin \left(\phi+\frac{\pi}{3}\right)\right]$ define the unit vectors of a hexagonal lattice ${ }^{69}$ with grid pattern orientation $\phi$ and $\operatorname{diag}(\cdot)$ produces a diagonal matrix from a vector input. Since most of the mass is associated with shorter transitions, in practice we approximate the full distribution with a finite number of periodic summations (i.e. ignore the tails; 5 cycles in our simulations; Fig. S1B).

The observation model defines the likelihood of the current environmental sensory inputs (i.e. the population vector of place cell firing $\boldsymbol{P}$, where $p_{i}(\overline{\boldsymbol{x}})=f\left(\overline{\boldsymbol{x}} \mid \boldsymbol{\mu}_{i}, \boldsymbol{\Sigma}_{P C}^{\mathrm{i}}\right)$ is the firing rate distribution of place cell $i$ over physical space $\overline{\boldsymbol{x}}$ ) given the predicted metric location $\boldsymbol{x}$, via a thresholded weighted sum: $H(\boldsymbol{P} \mid \boldsymbol{x})=\left[\sum_{i} B_{i}(\boldsymbol{x}) P_{i}\right]^{+}$. Here, $B_{i}(\boldsymbol{x})$ is the location distribution of landmark $i$ in metric space, which would be encoded biophysically in the learned $\left[N_{P} \times N_{G}\right]$ matrix $\boldsymbol{B}$ of synaptic weights from place to grid cells (i.e. the $g^{\text {th }}$ row and $i^{\text {th }}$ column of $\boldsymbol{B}$ is the distribution $B_{i}(\cdot)$ evaluated at the location of the $g^{t h}$ grid cell). The normalization constant $K=\int G(\boldsymbol{x}) d \boldsymbol{x}$ in Eq. 2 simply sums over the current grid cell activity and might biophysically be implemented by inhibitory interneurons. 


\section{Online learning of structural priors}

428 observation correction to $\boldsymbol{G}^{\prime}$ ).

\section{The offline probabilistic graphical model}

\section{Associative encoding in the hippocampus}

In the online model, the place-grid cell weight matrix $\boldsymbol{B}$ is learned using the $\mathrm{BCM}$ rule:

$$
\begin{aligned}
& \boldsymbol{B} \leftarrow \boldsymbol{B}+\tau_{P G} \boldsymbol{P}^{\top} \otimes\left[\boldsymbol{G}^{\prime} \odot\left(\boldsymbol{G}^{\prime}-\boldsymbol{\theta}\right)\right] \\
& \boldsymbol{\theta} \leftarrow \boldsymbol{\theta}+\tau_{\theta}[(\boldsymbol{G} \odot \boldsymbol{G})-\boldsymbol{\theta}]
\end{aligned}
$$

where $\tau_{P G}=1 e-4$ is the learning rate and $\boldsymbol{G}^{\prime}$ and $\boldsymbol{H}$ are column vectors whose elements are the transition and observation models estimated at the locations of a finite set of grid cell locations. $\odot$ is the element-wise (Hadamard) product between two vectors and $\otimes$ is their outer product. The sliding threshold $\boldsymbol{\theta} \in R^{1 \times N_{G}}$ provides adaptive synaptic normalization, where $\tau_{\theta} \approx 10 \tau_{P G}$. Learning takes place between the apriori distribution $\boldsymbol{G}^{\prime}$ and the current sensory observation $\boldsymbol{H}$ (i.e. before the

The pairwise potentials $\psi\left(\boldsymbol{b}_{i}-\boldsymbol{b}_{j}, \boldsymbol{\alpha} \mid \delta_{i j}\right)=\sum_{m, n=-\infty}^{\infty} \exp \left(-w_{i j}\left(\delta_{i j}-d_{m n}\left(\boldsymbol{b}_{i}-\boldsymbol{b}_{j}, \boldsymbol{\alpha}\right)\right)^{2}\right)$ penalize differences in the pairwise distances encoded by association $\delta_{i j}$, and those that would be computed by comparing their absolute encodings in metric space $d_{m n}(\cdot)$; i.e. they encourage a metric embedding that reflects the associative distance. The metric distance function $d_{m n}^{2}(\boldsymbol{x}, \boldsymbol{\alpha})=\left(\boldsymbol{x}+\boldsymbol{c}_{m n}\right)^{\mathrm{T}} \boldsymbol{\alpha}(\boldsymbol{x}+$ $\left.\boldsymbol{c}_{m n}\right)$ defines the pairwise distance between the encoding of locations $i$ and $j$ in $\mathrm{mEC}$ in metric space, and is dependent on the gain factor $\boldsymbol{\alpha}$ of the transition model. Pairwise measurements are assumed to have confidence $w_{i j}$ (inverse variance) that increases with decreasing inferred distance (i.e. $w_{i j}=$ $\left.1 /\left(\sigma_{P C}+\sigma_{P I} \delta_{i j}\right)\right)$. The transition model gain is assumed to have a Gaussian prior $p(\boldsymbol{\alpha})=$ $\exp \left(-w_{\alpha}\left(\boldsymbol{\alpha}-\boldsymbol{\alpha}_{\mathbf{0}}\right)^{\mathrm{T}}\left(\boldsymbol{\alpha}-\boldsymbol{\alpha}_{\mathbf{0}}\right)\right)$, the $w_{\alpha}$ term representing the confidence in the prior gain value $\boldsymbol{\alpha}_{0}$. The periodic offset term $\boldsymbol{c}_{m n}$ is the same as defined for the transition model.

The associative distances are recovered from the $\left[N_{P} \times N_{p}\right]$ synaptic weights in CA3 $A$. Under a random-walk behavioural trajectory, the simple modified Hebbian learning rule:

$$
\boldsymbol{A} \leftarrow \boldsymbol{A}+\tau_{P P}\left[\boldsymbol{P} \boldsymbol{P}^{\top}-\boldsymbol{A} \odot \boldsymbol{A}\right]
$$


where $\odot$ is the element-wise (Hadamard) product and $\tau_{P P}$ the learning rate. The synaptic weights can be shown to converge to $A_{i j}=\sqrt{\left\langle P_{i}(t) P_{j}(t)\right\rangle}$, the square-root of the correlation between the firing rates of two $\mathrm{PCs}^{70}$. Where place fields have uniform receptive field widths $\left(\sigma_{P C}\right)$ and peak firing rates, the Euclidean distance between place fields $i$ and $j$ can be inferred via the simple transformation ${ }^{46}$ :

$$
\delta_{i j}^{2}=-\log \left(A_{i j}\right)=\frac{\left(\mu_{i}-\mu_{j}\right)^{2}}{2 \sigma_{P C}^{2}}
$$

The recovered distance is therefore scaled by the receptive fields' variance (the Bhattacharyya distance $^{46}$ ), and so relates to 'discriminability' (Fig. 1D). CA3 synapses effectively average over multiple pairwise measurements. By assuming that noise in the pairwise distance measurements scale linearly with distance, both the mean and variance of the Gaussian describing this distribution is efficiently encoded in a single PC-PC synapse.

\section{Simplified analysis of the probabilistic graphical model likelihood}

To characterize model predictions in the environmental rescaling ${ }^{71}$ and gain change ${ }^{7}$ experiments, we studied a reduced version of the full graphical model (Eq. 3; see Supplementary Methods for full derivation). In $1 \mathrm{D}$, given a linear observation model $x=H\left(x^{\prime}\right)=K_{1}+K x^{\prime}$ and a large number of evenly spaced place fields, Eq. 3 simplifies to (see Supplementary Methods):

$$
-\log L=\ell \widetilde{\propto}\left(1 / \alpha^{\text {Visual }}-K / \alpha\right)^{2}+\mathrm{P}_{\mathrm{C}}\left(K-K^{0}\right)^{2}+\mathrm{P}_{\mathrm{C}} \cdot \mathrm{T}_{\mathrm{C}}\left(1 / \alpha-1 / \alpha^{0}\right)^{2}
$$

which can be solved analytically. A similar reduction was applied to the $2 \mathrm{D}$ case when considering differential shifts in grid and place fields ${ }^{23}$.

\section{Belief propagation for offline inference}

Belief propagation ${ }^{48}$ is an iterative, two-stage local message-passing scheme in which, at each iteration $n$, a feature node (i.e. a place cell) first updates its own belief (connections to the grid cells) $B_{i}^{(n)}$ by integrating messages from connected nodes $j \in \Gamma_{i}$ with its own prior belief $B_{i}^{(0)}$ (Fig. 5C):

$$
B_{i}^{(n)}\left(\boldsymbol{b}_{i}\right) \propto B_{i}^{(0)}\left(\boldsymbol{b}_{i}\right) \prod_{j \in \Gamma_{i}} m_{j \rightarrow i}^{(n)}\left(\boldsymbol{b}_{i}\right)
$$


474 The message from node $j$ to node $i\left(m_{j \rightarrow i}\right)$ communicates its belief over the distribution of the

475 locations of place cell $i$ in grid cell space, conditioned on its own location distribution:

$$
m_{j \rightarrow i}^{(n+1)}\left(\boldsymbol{b}_{i}\right) \propto \int \psi\left(\boldsymbol{b}_{j}-\boldsymbol{b}_{i}, \boldsymbol{\alpha} \mid \delta_{j i}\right) \cdot\left[\frac{B_{j}^{(n)}\left(\boldsymbol{b}_{j}\right)}{m_{i \rightarrow j}^{(n)}\left(\boldsymbol{b}_{j}\right)}\right] d \boldsymbol{b}_{j}
$$

where the pairwise potentials $\psi(\cdot)$ are the same as those described in the full likelihood function (Eq.

3). The graph converges when new messages cease to change the beliefs of their recipient nodes.

\section{Scheduled message passing on the place cell graph}

'Synchronous' belief propagation computes belief updates for each step before broadcasting all new between the node's previous $\boldsymbol{B}_{i}^{(n-1)}$ and updated $\boldsymbol{B}_{i}^{(n)}$ beliefs is defined as:

where $\mathbb{J} \mathbb{S}$ is the Jensen-Shannon (symmetric K-L) divergence, where:

$$
\mathcal{T}_{i}^{n}=\mathbb{J} \mathbb{S}\left(B_{i}^{(n)} \| B_{i}^{(n-1)}\right)=\frac{1}{2}\left[\mathbb{K} \mathbb{L}\left(B_{i}^{(n)} \| m\right)+\mathbb{K} \mathbb{L}\left(B_{i}^{(n-1)} \| m\right)\right]
$$

$$
m=\frac{1}{2}\left(B_{i}^{(n)}+B_{i}^{(n-1)}\right)
$$

$$
\mathbb{K L}(p \| q)=-\int p(x) \cdot \log \left(\frac{q(x)}{p(x)}\right) d x
$$

When the message tension is below a pre-defined threshold $\mathcal{T}_{\min }$, a node has converged and ceases to broadcast new messages. This mechanism is similar to the prediction error between transition and observation models used to trigger offline inference, with the exception that it uses the symmetric divergence measure $\mathbb{J S}$ rather than $\mathbb{K L}$.

\section{Traveling waves in neural media}

In simulations, the traveling waves in mEC are simulated explicitly by calculating the true messages 
495

496

497

(Fig. 7), messages were approximated as waves propagating radially from an initial stimulation on the mEC sheet using a modified mechanical wave model, as used to describe oscillations in water:

$$
\frac{d v^{2}}{d t}=c^{2} \nabla^{\prime 2} \cdot[v]^{+}
$$

where $c$ is the speed of wave propagation, $[\cdot]^{+}$is a threshold linear activation function, and the modified spatial Laplacian operator $\nabla^{\prime}$ is a symmetric $2 \mathrm{D}$ Gaussian filter with variance equal to the PI noise (see Supplementary Methods for extended discussion).

\section{Acknowledgements}

We thank the groups of Caswell Barry and Francesca Cacucci for use of their data and Martin Pearson (UWE and Bristol Robotics Lab), Dan Bush, Andrej Bicanski and Tim Behrens for valuable theoretical discussions.

We acknowledge funding by the ERC Advanced grant NEUROMEM, the Wellcome Trust and the European Union's Horizon 2020 research and innovation programme under grant agreement No. 785907 Human Brain Project SGA2. The authors declare no competing financial interests.

\section{References}

1. Burak, Y. \& Fiete, I. R. Accurate path integration in continuous attractor network models of grid cells. PLoS computational biology 5, e1000291 (2009).

2. Fuhs, M. C. \& Touretzky. A Spin Glass Model of Path Integration in Rat Medial Entorhinal Cortex. Journal of Neuroscience 26, 4266-4276 (2006).

3. McNaughton, B. L., Battaglia, F. P., Jensen, O., Moser, E. I. \& Moser, M.-B. Path integration and the neural basis of the 'cognitive map'. Nature Reviews Neuroscience 7, 663-678 (2006).

4. Lever, C., Burton, S., Jeewajee, A., O'Keefe, J. \& Burgess, N. Boundary Vector Cells in the Subiculum of the Hippocampal Formation. Journal of Neuroscience 29, 9771-9777 (2009).

5. Hartley, T., Burgess, N., Lever, C., Cacucci, F. \& O'keefe, J. Modeling place fields in terms of the cortical inputs to the hippocampus. Hippocampus 10, 369-379 (2000). 
6. O’Keefe, J. \& Burgess, N. Dual phase and rate coding in hippocampal place cells: Theoretical significance and relationship to entorhinal grid cells. Hippocampus 15, 853-866 (2005).

7. Chen, G., Lu, Y., King, J. A., Cacucci, F. \& Burgess, N. Differential influences of environment and self-motion on place and grid cell firing. Nature communications 10, 630 (2019).

8. Hardcastle, K., Ganguli, S. \& Giocomo, L. M. Environmental Boundaries as an Error Correction Mechanism for Grid Cells. Neuron (2015) doi:10.1016/j.neuron.2015.03.039.

9. Keinath, A. T., Epstein, R. A. \& Balasubramanian, V. Environmental deformations dynamically shift the grid cell spatial metric. eLife 7, e38169 (2018).

10. Durrant-Whyte, H. \& Bailey, T. Simultaneous localization and mapping: part I. IEEE Robotics \& Automation Magazine 13, 99-110 (2006).

11. Milford, M. J., Wyeth, G. F. \& Prasser, D. RatSLAM: a hippocampal model for simultaneous localization and mapping. in Robotics and Automation, 2004. Proceedings. ICRA'04. 2004 IEEE International Conference on vol. 1 403-408 (IEEE, 2004).

12. Guo, W., Zhang, J. J., Newman, J. \& Wilson, M. A. Latent learning drives sleep-dependent plasticity in distinct CA1 subpopulations. bioRxiv (2020).

13. Ocko, S. A., Hardcastle, K., Giocomo, L. M. \& Ganguli, S. Emergent elasticity in the neural code for space. Proceedings of the National Academy of Sciences 115, E11798-E11806 (2018).

14. Penny, W. D., Zeidman, P. \& Burgess, N. Forward and backward inference in spatial cognition. PLoS computational biology 9, e1003383 (2013).

15. Evans, T. \& Burgess, N. Coordinated hippocampal-entorhinal replay as structural inference. in Advances in Neural Information Processing Systems (2019).

16. Diba, K. \& Buzsáki, G. Forward and reverse hippocampal place-cell sequences during ripples. Nature Neuroscience 10, 1241-1242 (2007).

17. Foster, D. J. \& Wilson, M. A. Reverse replay of behavioural sequences in hippocampal place cells during the awake state. Nature 440, 680-683 (2006).

18. Ólafsdóttir, H. F., Carpenter, F. \& Barry, C. Coordinated grid and place cell replay during rest. Nature Neuroscience 19, 792-794 (2016). 
19. O’Neill, J., Boccara, C., Stella, F., Schoenenberger, P. \& Csicsvari, J. Superficial layers of the medial entorhinal cortex replay independently of the hippocampus. Science 355, 184-188 (2017).

20. Wilson, M. A. \& McNaughton, B. L. Dynamics of the hippocampal ensemble code for space. Science 261, 1055-1058 (1993).

21. Barry, C., Hayman, R., Burgess, N. \& Jeffery, K. J. Experience-dependent rescaling of entorhinal grids. Nature Neuroscience 10, 682-684 (2007).

22. Barry, C., Ginzberg, L. L., O’Keefe, J. \& Burgess, N. Grid cell firing patterns signal environmental novelty by expansion. Proceedings of the National Academy of Sciences 109, $17687-17692(2012)$.

23. Krupic, J., Bauza, M., Burton, S. \& O’Keefe, J. Local transformations of the hippocampal cognitive map. Science 359, 1143-1146 (2018).

24. Stensola, H. et al. The entorhinal grid map is discretized. Nature 492, 72-78 (2012).

25. Butler, W. N., Hardcastle, K. \& Giocomo, L. M. Remembered reward locations restructure entorhinal spatial maps. Science 363, 1447-1452 (2019).

26. Hägglund, M., Mørreaunet, M., Moser, M.-B. \& Moser, E. I. Grid-Cell Distortion along Geometric Borders. Current Biology (2019) doi:10.1016/j.cub.2019.01.074.

27. Hollup, S. A., Molden, S., Donnett, J. G., Moser, M.-B. \& Moser, E. I. Accumulation of hippocampal place fields at the goal location in an annular watermaze task. Journal of Neuroscience 21, 1635-1644 (2001).

28. Munn, R. G. K., Mallory, C. S., Hardcastle, K., Chetkovich, D. M. \& Giocomo, L. M. Entorhinal velocity signals reflect environmental geometry. bioRxiv (2019) doi:10.1101/671222.

29. Stensola, T., Stensola, H., Moser, M.-B. \& Moser, E. I. Shearing-induced asymmetry in entorhinal grid cells. Nature 518, 207-212 (2015).

30. Hafting, T., Fyhn, M., Molden, S., Moser, M.-B. \& Moser, E. I. Microstructure of a spatial map in the entorhinal cortex. Nature 436, 801-806 (2005).

31. Fiete, I. R., Burak, Y. \& Brookings, T. What Grid Cells Convey about Rat Location. Journal of Neuroscience 28, 6858-6871 (2008). 
32. Mathis, A., Herz, A. V. \& Stemmler, M. Optimal population codes for space: grid cells outperform place cells. Neural computation 24, 2280-2317 (2012).

33. Stemmler, M., Mathis, A. \& Herz, A. V. M. Connecting multiple spatial scales to decode the population activity of grid cells. Science Advances 1, e1500816-e1500816 (2015).

34. Guanella, A., Kiper, D. \& Verschure, P. A model of grid cells based on a twisted torus topology. International journal of neural systems 17, 231-240 (2007).

35. Stachenfeld, K. L., Botvinick, M. M. \& Gershman, S. J. The hippocampus as a predictive map. Nature Neuroscience (2017) doi:10.1038/nn.4650.

36. Whittington, J., Muller, T., Mark, S., Barry, C. \& Behrens, T. Generalisation of structural knowledge in the hippocampal-entorhinal system. in Advances in Neural Information Processing Systems 31 (eds. Bengio, S. et al.) 8484-8495 (Curran Associates, Inc., 2018).

37. Widloski, J. \& Fiete, I. R. A Model of Grid Cell Development through Spatial Exploration and Spike Time-Dependent Plasticity. Neuron 83, 481-495 (2014).

38. Kropff, E., Carmichael, J. E., Moser, M.-B. \& Moser, E. I. Speed cells in the medial entorhinal cortex. Nature 523, 419-424 (2015).

39. Munn, R. G. K., Mallory, C. S., Hardcastle, K., Chetkovich, D. M. \& Giocomo, L. M. Entorhinal velocity signals reflect environmental geometry. Nat Neurosci (2020) doi:10.1038/s41593-0190562-5.

40. Milford, M. J. \& Wyeth, G. F. Mapping a suburb with a single camera using a biologically inspired SLAM system. IEEE Transactions on Robotics 24, 1038-1053 (2008).

41. Muller, R. U., Stead, M. \& Pach, J. The hippocampus as a cognitive graph. The Journal of general physiology 107, 663-694 (1996).

42. Burgess, N. \& O'Keefe, J. Neuronal computations underlying the firing of place cells and their role in navigation. Hippocampus 6, 749-762 (1996).

43. Campbell, M. G. et al. Principles governing the integration of landmark and self-motion cues in entorhinal cortical codes for navigation. Nature neuroscience 21, 1096 (2018).

44. Krupic, J., Bauza, M., Burton, S., Barry, C. \& O'Keefe, J. Grid cell symmetry is shaped by environmental geometry. Nature 518, 232-235 (2015). 
601

602

603

604

605

606

607

608

609

610

611

612

613

614

615

616

617

618

619

620

621

622

45. Hägglund, M. \& Mørreaunet, M. Grid pattern development, distortions and topological defects may depend on distributed anchoring. BioRxiv 830158 (2019).

46. Bhattacharyya, A. On a measure of divergence between two statistical populations defined by their probability distributions. Bull. Calcutta Math. Soc. 35, 99-109 (1943).

47. Tanni, S. \& Barry, C. Relationship between place cell recruitment and the spatial scale of openfield environments revealed using a novel scalable recording apparatus. in Society for Neuroscience Program No. 689.18. (Society for Neuroscience, 2018).

48. Pearl, J. Reverend Bayes on inference engines: A distributed hierarchical approach. in National Conference on Artificial Intelligence 133--136 (AIII Press, 1982).

49. Elidan, G., McGraw, I. \& Koller, D. Residual belief propagation: Informed scheduling for asynchronous message passing. arXiv preprint arXiv:1206.6837 (2012).

50. Davidson, T. J., Kloosterman, F. \& Wilson, M. A. Hippocampal Replay of Extended Experience. Neuron 63, 497-507 (2009).

51. Stella, F., Baracskay, P., O’Neill, J. \& Csicsvari, J. Hippocampal Reactivation of Random Trajectories Resembling Brownian Diffusion. Neuron (2019) doi:10.1016/j.neuron.2019.01.052.

52. Bellmund, J. L. et al. Deforming the metric of cognitive maps distorts memory. Nature human behaviour 1-12 (2019).

53. Boccara, C. N., Nardin, M., Stella, F., O’Neill, J. \& Csicsvari, J. The entorhinal cognitive map is attracted to goals. Science 363, 1443-1447 (2019).

54. Nakazawa, K. et al. Requirement for hippocampal CA3 NMDA receptors in associative memory recall. Science 297, 211-218 (2002).

55. Dordek, Y., Soudry, D., Meir, R. \& Derdikman, D. Extracting grid cell characteristics from place cell inputs using non-negative principal component analysis. eLife 5, (2016).

56. Banino, A. et al. Vector-based navigation using grid-like representations in artificial agents. Nature 557, 429 (2018).

57. Carpenter, F., Manson, D., Jeffery, K., Burgess, N. \& Barry, C. Grid Cells Form a Global Representation of Connected Environments. Current Biology 1-7 (2015) doi:10.1016/j.cub.2015.02.037. 
58. Lee, A. K. \& Wilson, M. A. Memory of sequential experience in the hippocampus during slow wave sleep. Neuron 36, 1183-1194 (2002).

59. Skaggs, W. E. \& McNaughton, B. L. Replay of neuronal firing sequences in rat hippocampus during sleep following spatial experience. Science 271, 1870-1873 (1996).

60. Wilson, M. A. \& McNaughton, B. L. Reactivation of hippocampal ensemble memories during sleep. Science 265, 676-679 (1994).

61. Buzsáki, G., Vanderwolf, C. H. \& others. Cellular bases of hippocampal EEG in the behaving rat. Brain Research Reviews 6, 139-171 (1983).

62. O'Keefe, J. Place units in the hippocampus of the freely moving rat. Experimental neurology 51, 78-109 (1976).

63. Bicanski, A. \& Burgess, N. A neural-level model of spatial memory and imagery. eLife 7, e33752 (2018).

64. Mattar, M. G. \& Daw, N. D. Prioritized memory access explains planning and hippocampal replay. (2018) doi:10.1101/225664.

65. Momennejad, I., Otto, A. R., Daw, N. D. \& Norman, K. A. Offline replay supports planning in human reinforcement learning. Elife 7, e32548 (2018).

66. Hasselmo, M. E. Neuronal rebound spiking, resonance frequency and theta cycle skipping may contribute to grid cell firing in medial entorhinal cortex. Philosophical Transactions of the Royal Society of London B: Biological Sciences 369, 20120523 (2014).

67. Muller, L., Chavane, F., Reynolds, J. \& Sejnowski, T. J. Cortical travelling waves: mechanisms and computational principles. Nature Reviews Neuroscience (2018).

68. Yamamoto, J. \& Tonegawa, S. Direct medial entorhinal cortex input to hippocampal CA1 is crucial for extended quiet awake replay. Neuron 96, 217-227 (2017).

69. Mathis, A., Stemmler, M. B. \& Herz, A. V. Probable nature of higher-dimensional symmetries underlying mammalian grid-cell activity patterns. Elife 4, e05979 (2015).

70. Dayan, P. \& Abbott, L. F. Theoretical neuroscience. (Cambridge, MA: MIT Press, 2001).

71. Barry, C. \& Burgess, N. Learning in a geometric model of place cell firing. Hippocampus 17, $786-800$ (2007). 
bioRxiv preprint doi: https://doi.org/10.1101/2020.08.07.241547; this version posted August 10, 2020. The copyright holder for this preprint (which was not certified by peer review) is the author/funder. All rights reserved. No reuse allowed without permission. 
658 Figure 1. Online+offline localization and mapping. A) Illustration of recursive Bayesian

659 integration. A probability distribution over current location $(G(\boldsymbol{x}))$, represented by grid cell

660 firing, is updated according to self-motion via the transition model $\left(\mathrm{T}\left(\mathbf{x}, \mathbf{x}^{\prime} \mid \widehat{\mathbf{u}}, \boldsymbol{\alpha}\right)\right)$ then refined

661 by environmental inputs via the observation model $\left(H\left(\boldsymbol{P}_{t} \mid \boldsymbol{x}_{t}\right)\right)$. C) Estimates based on the

662 integration of noisy self-motion and environmental inputs may be stable, as shown in

663 simulated grid cell firing rate maps (right), despite instability when using only self-motion

664 (left) or environmental inputs after brief initial exploration (middle; numbers show gridness

score). C) Simulated grid cells exhibit spatially offset grid-like firing patterns, due to toroidal connectivity, despite the absence of attractor dynamics. Right shows histogram of spatial

667 phases. D) Inferred pairwise distances D are a function of the 'overlap' between place fields.

668 E) Pairwise distances can be used to infer the structure of the world (the mapping of place

669 fields onto the grid map). F) Given noisy initial priors ("Initial"), structural encodings are

670 modified to reflect pairwise associative measurements. Inferred structure is sensitive to the topology of the environment (cf. "Ring" and "Broken Ring"). G) Illustration of the prediction error mechanism used to arbitrate between the online and offline systems (blue bars show prediction error $\varepsilon, \varepsilon_{0}$ is the minimum prediction error needed to trigger offline inference).

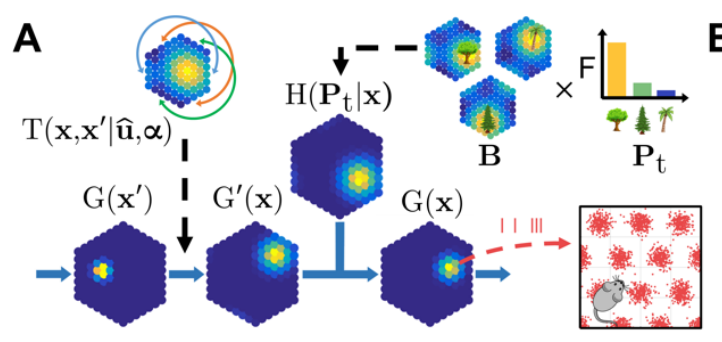

B

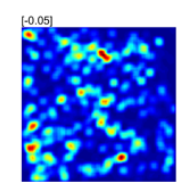

Transition model Observation model estimate

E

D

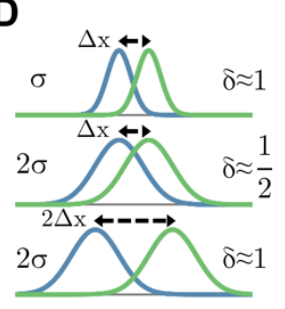

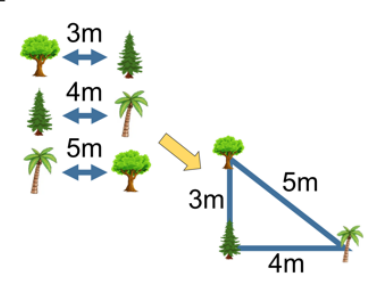

$\mathbf{F}$

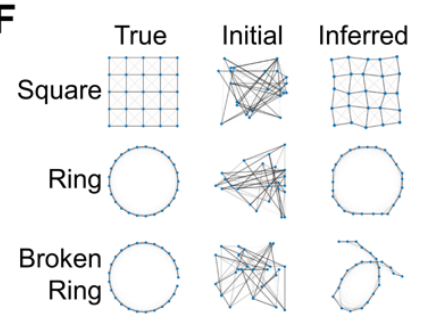

C
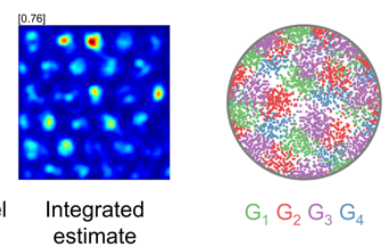

$\mathbf{G}$

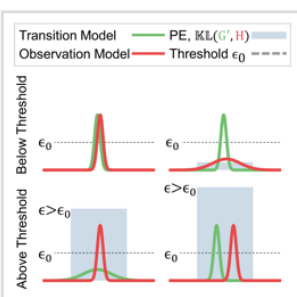


bioRxiv preprint doi: https://doi.org/10.1101/2020.08.07.241547; this version posted August 10, 2020. The copyright holder for this preprint (which was not certified by peer review) is the author/funder. All rights reserved. No reuse allowed without permission. 
677 Figure 2. Minimizing prediction errors in the offline system: grid rescaling and direction678 dependent offsets under manipulations of environmental size or VR gain. A) During online 679 spatial localization, the observation model estimates location in metric space (activity on the 680 grid sheet) via inputs from place cells driven by environmental features (red curve), the transition model updates the previous estimate (dashed blue curve) according to self-motion (green curve), producing a combined estimate (blue curve). Manipulations of the environment cause the predictions from both models to diverge. One way to minimize these prediction errors is to modify the observation model by changing the connection weights from place to grid cells (i.e. the mapping between environmental observations to estimated location in grid space). An alternative is to modify the transition model to reflect the observation model estimate (e.g. varying the 'gain' mapping self-motion to grid space). The degree to which both are modified is controlled by the relative strength of their respective prior confidences $\left(\mathrm{T}_{\mathrm{C}}\right)$. B) Joint optimization of the observation and transition models predict partial rescaling of grid patterns in response to increase in the VR gain (i.e. the rate of visual movement in response to physical movement on the ball; below) or compression of a real environment (above). When the system is confident in its self-motion ( $\mathrm{T}_{\mathrm{C}} \rightarrow \infty$, green), the observation model is modified to match the transition model (no change in grid scale plotted in the real or visual VR environment). When the system is confident in its environmental inputs $\left(\mathrm{T}_{\mathrm{C}} \rightarrow 0\right.$, red), the transition model adapts and grid scale follows the environmental change. Balanced model confidence produces intermediate rescaling (blue). C) Change in observed grid scale (grid pattern plotted against self-motion) depends on the transition confidence values $\mathrm{T}_{\mathrm{C}}$. $\mathrm{X}$-axis shows $\mathrm{VR}$ gain change or environmental compression, where $\alpha^{\text {Visual }}=1$ is a control trial. Y-axis shows observed change in grid scale (see Methods). Data points show corresponding values from Barry et al. (2007), Chen et al. (2019) and Campbell et al., (2019), which suggest an approximately equal weighting of transition and observation model priors for both gain decreases and increase trials $\left(T_{C} \approx 1\right)$. $\left.D\right)$ Firing rate map of a grid cell on the linear track in control (solid line) and VR gain decrease trials $\left(\alpha^{\text {Visual }}=0.7\right)$, 
704 plotted in the visual VR environment. In the gain decrease condition, the grid fields are stably

705 shifted to fire earlier when running left (dashed line) or right (dotted line), i.e. towards the

706 location indicated by the transition model from that indicated by vision. E) The direction-

707 dependent shift in estimated location in the grid module in a VR gain decrease trial

$708\left(\alpha^{\text {Visual }}=2 / 3\right)$ stabilises at a fixed distance. Sequence of eight updates of estimated location

709 on the grid module $G(x)$ when running to the right in a VR visual gain decrease trial, colours

710 as in A. The transition model (green) predicts a location ahead of that from the observation

711 model on the grid sheet (red; driven by visual input) because of the visual gain decrease.

712 Combining these estimates produces an intermediate distribution (blue). At each new

713 update, the prediction from the transition model builds on the shift of the previous combined

714 estimate (not the previous transition model estimate) so that the distance between the

715 observation model estimate and the combined estimate stabilizes at a fixed value, producing

716 a fixed direction-dependent offset of the grid patterns in environmental coordinates (see the

717 differences in the location estimates between models, Inset). 
A

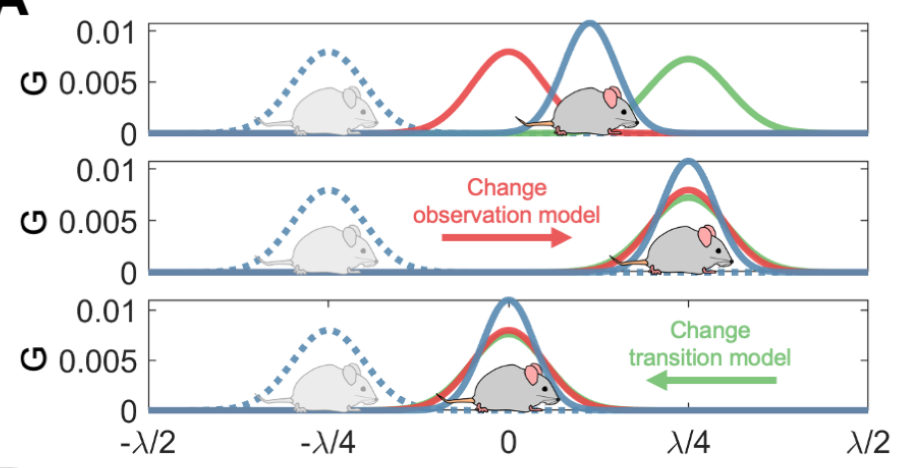

B

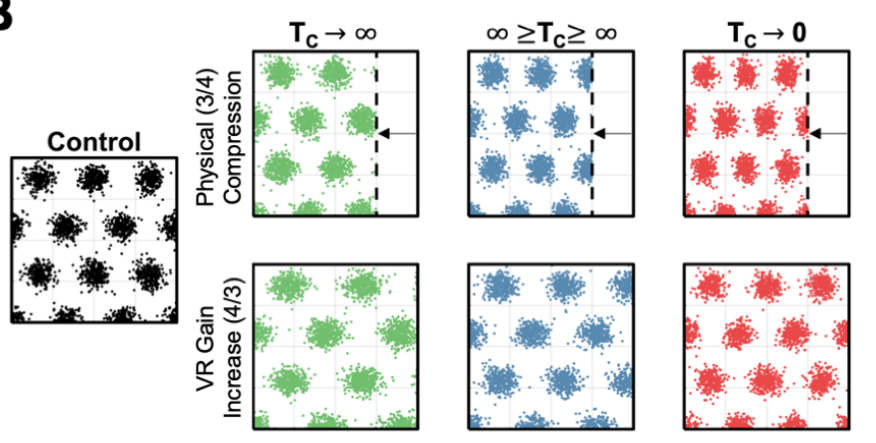

C

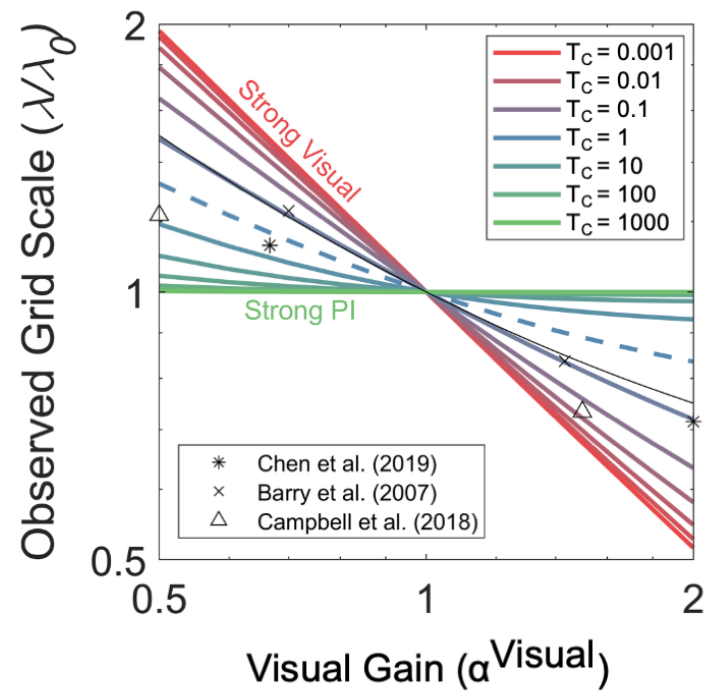

$\mathbf{E}$
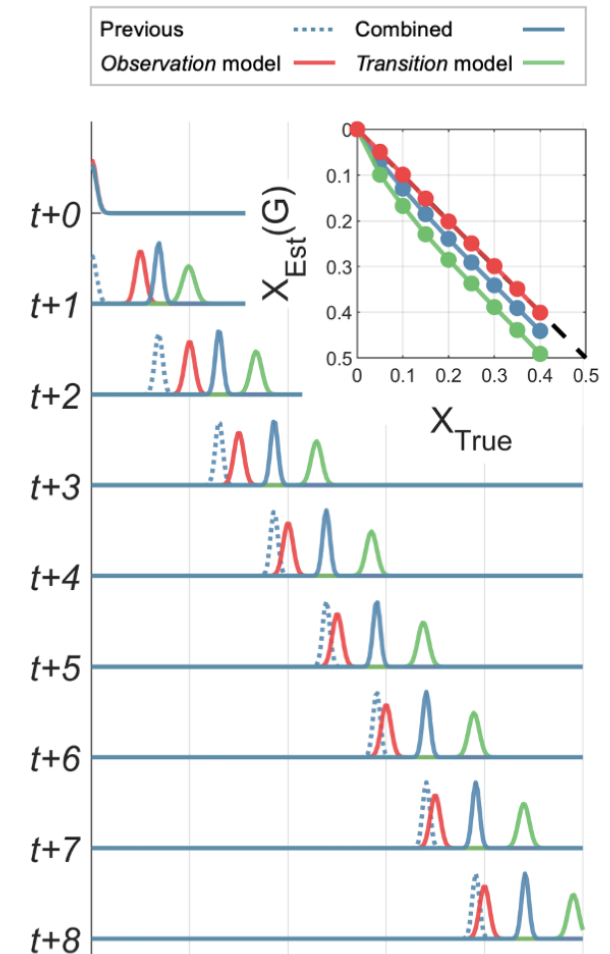

D

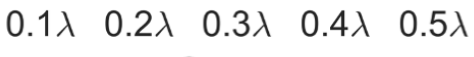

$\mathrm{G}$

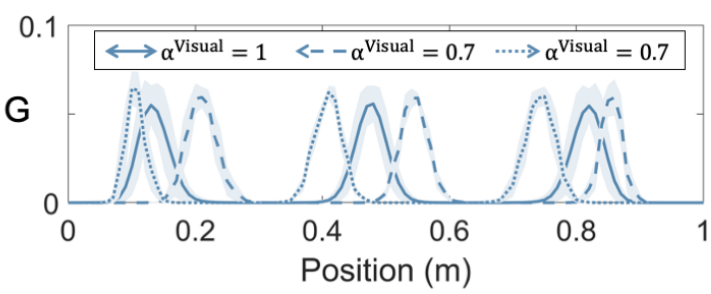


720 Figure 3. Effects of local deformation of a rectangular environment on grid patterns following

721 offline inference (Krupic et al., 2018). Dots indicate place/grid field locations, arrows indicate

722 shifts before/after offline structural optimization and coloured hexagons indicate magnitude

723 of shift vector. Grid patterns show estimate generated by the observation model (weighted

724 place cell activity). A) Place field shifts were measured from Krupic et al. (2018) and

725 interpolated and smoothed. B-C) Place field shifts cause immediate prediction errors (PE)

726 between the new pairwise place field distances and the distances between their

727 corresponding grid locations. If the observation / transition model priors are weak $\left(\mathrm{P}_{\mathrm{C}} \rightarrow 0\right)$,

728 PEs are eliminated during offline inference by updating the observation model (top row;

729 unlike in Fig. 4B, modifying the global gain did not remove the effect of local distortions).

730 Complete adaptation in the observation model leads to an unperturbed grid pattern, i.e. grid

731 fields will not shift. Alternatively, strong model priors $\left(\mathrm{P}_{\mathrm{C}} \rightarrow \infty\right)$ prevent adaptation to new

732 environmental inputs, leading to distorted grid patterns when driven purely by the observation model, whose field shifts match those of the place cells (bottom row). When the model priors are balanced against the new pairwise observations, the observation model is partially adjusted, producing partial grid field shifts (which are smaller than those of the place fields; middle row). Partial (middle) or no (bottom) adjustment to new observations preserve mismatches between the transition and observation models, which would result in directiondependent offsets (see Figure 4E) and irregular firing patterns (see Figure S2D). NB the confidence in the prior model may depend on location, e.g. if there is strong anchoring to the wall prior connections from place cells with fields near the wall may be stronger than those with fields further away. D) Comparison of experimentally observed place and grid field shifts

742 with simulation corresponding to the middle row of B-C. 


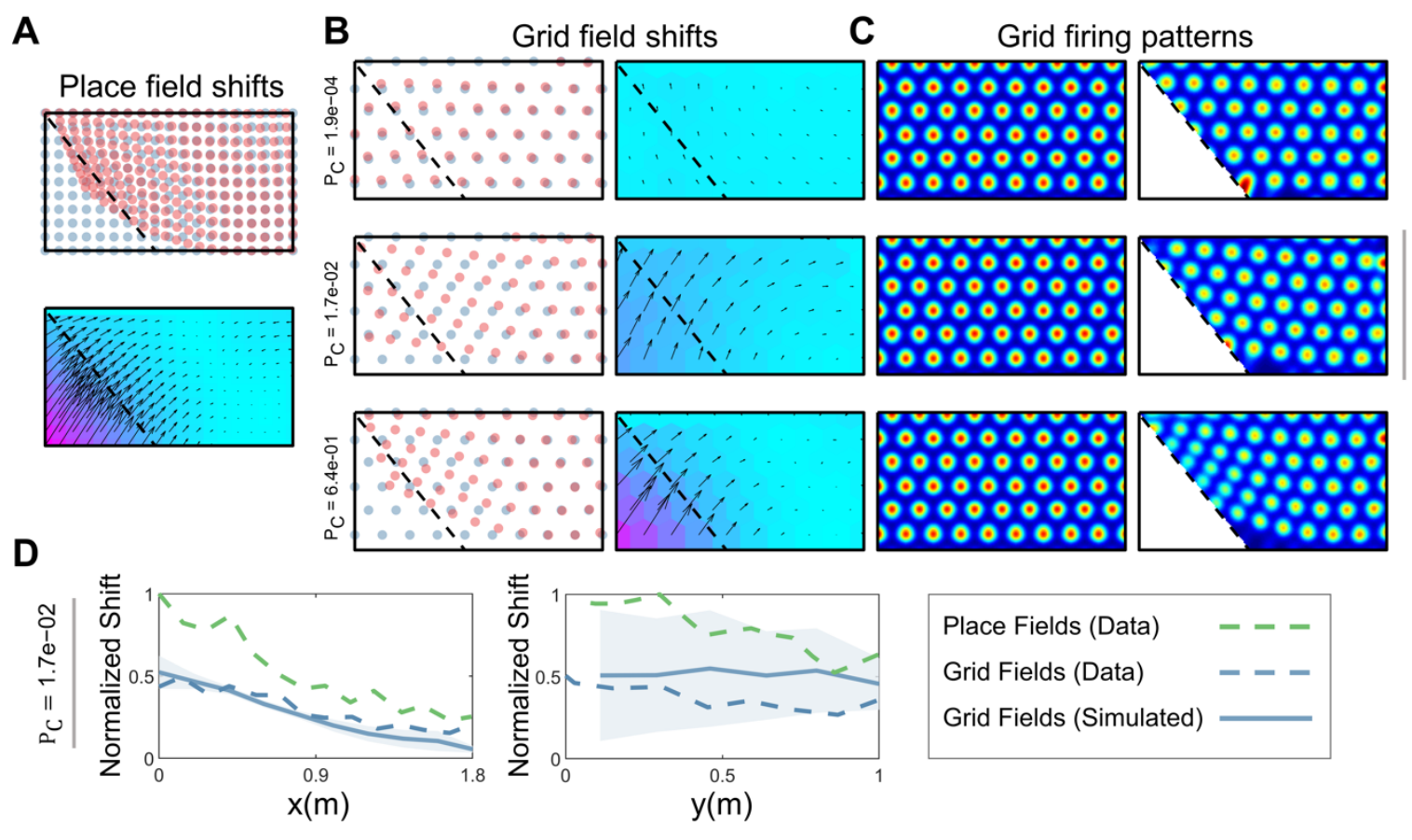


745 Figure 4. Distortions to grid patterns caused by inhomogeneous environmental input during

746 offline inference. A) Simulated variation in place field shape due to proximity to boundaries

747 (above), or inhomogeneous sampling of locations (Hagglund et al,. 2019; below) results in

748 distortions to the inferred pairwise distances in CA3 (B). These distortions lead to distortions

749 to grid scale due to adjustment of the observation model during offline inference (C-D).

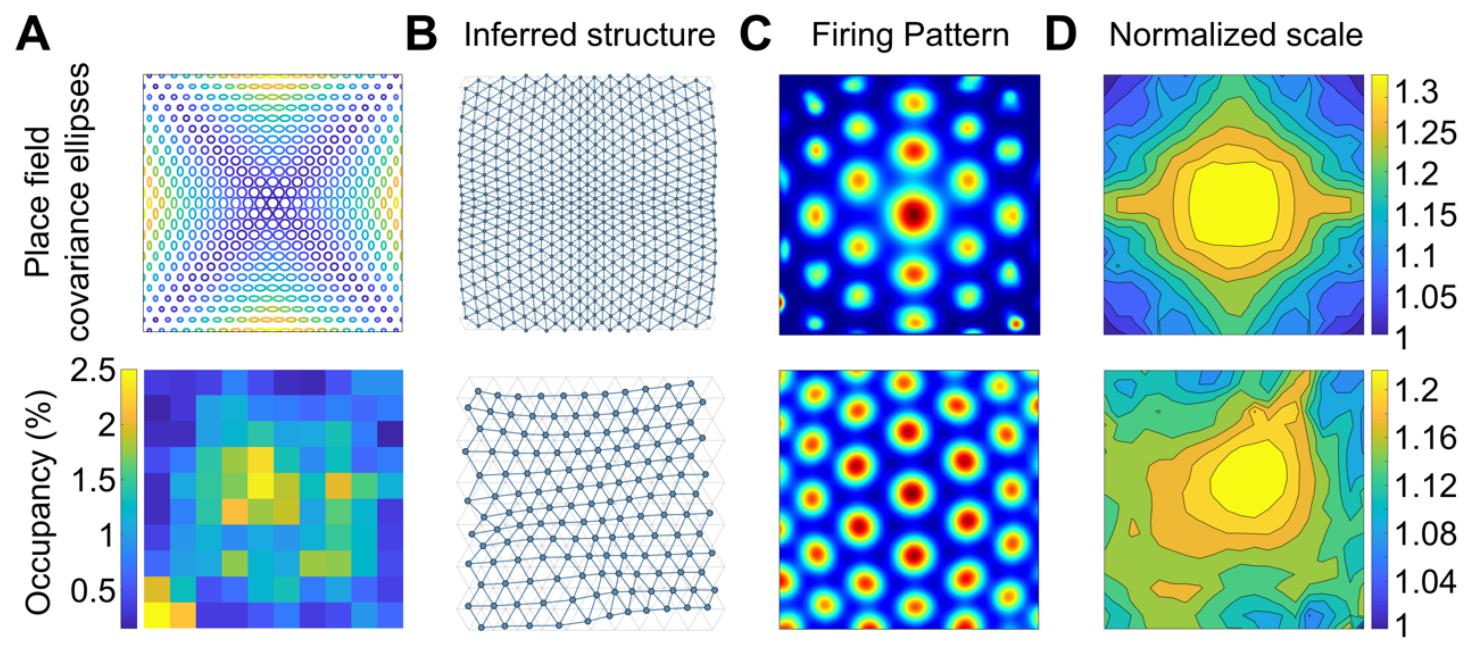


752 Figure 5. Illustration of the dual-systems model: prediction errors and replay in the loop-

753 closure task (see also Supplementary Video 1). A) Place cell - grid cell (PC-GC) connection

754 weights (above) as agent runs around a circular track for the first time (below, GC activity

755 and the input from PCs to GCs, both shown on topographically organised sheet of cells,

756 inset). i) Confidence in the initial location is high, such that coactive GC and PC fields form

757 strong associations (the GC firing distribution is peaked and the inputs from place cells with

758 fields at the beginning of the track are strong). ii) The agent navigates around the track,

759 accumulating self-motion error, leading to diffuse GC firing. iii) Prediction errors (PE) on lap

760 completion (when the initially learned precise PC input arrives) triggers an offline inference

761 event (see main text and Supp. Video 1 for details). iv) On subsequent laps of the track, PC-

762 GC weights are sharply tuned following offline inference, allowing effective localization. B)

763 PE is reduced on completion of subsequent laps due to alignment of the transition and observation models (i.e. environmental inputs and self-motion updating of GC activity coincide). C) Illustration of belief propagation. Place cell A receives messages from PCs B and $\mathrm{C}$. Messages take the form of rings, describing a preferred distance about the current locations of $B$ and $C$ with variance reflecting the confidence in the message (the variance of pairwise distance estimates with Gaussian noise). The intersection of the messages uniquely determines the location of A over time. NB A will also be broadcasting messages back to B and C. D) True structure (blue), structure encoded by noisy path integration (left, red; i.e. the location of the peaks of the weights from each place cell to the grid cell sheet) and structure inferred after loop-closure (right, green). E) Offline inference allows one-shot learning when compared to the online system alone. 

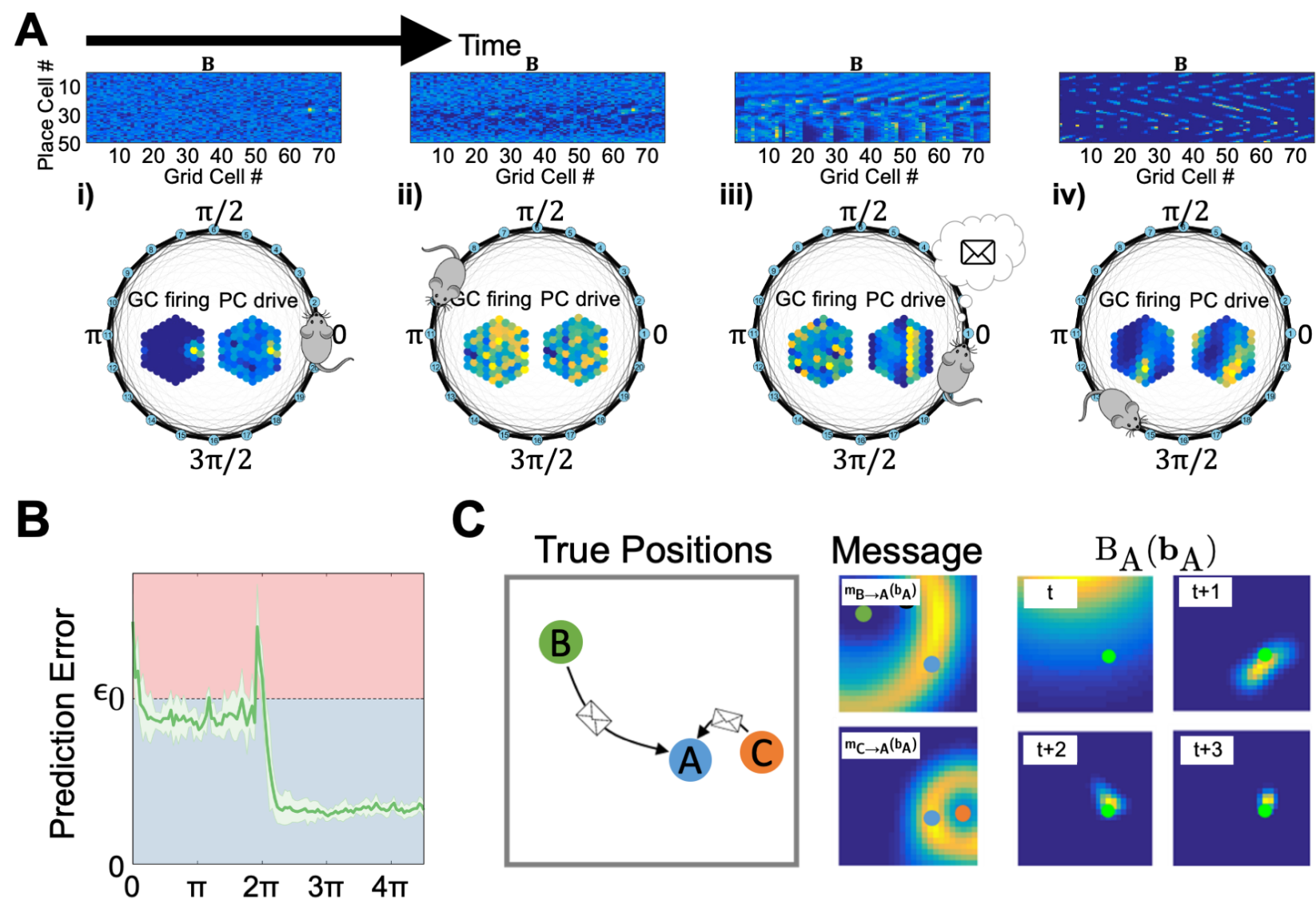

D
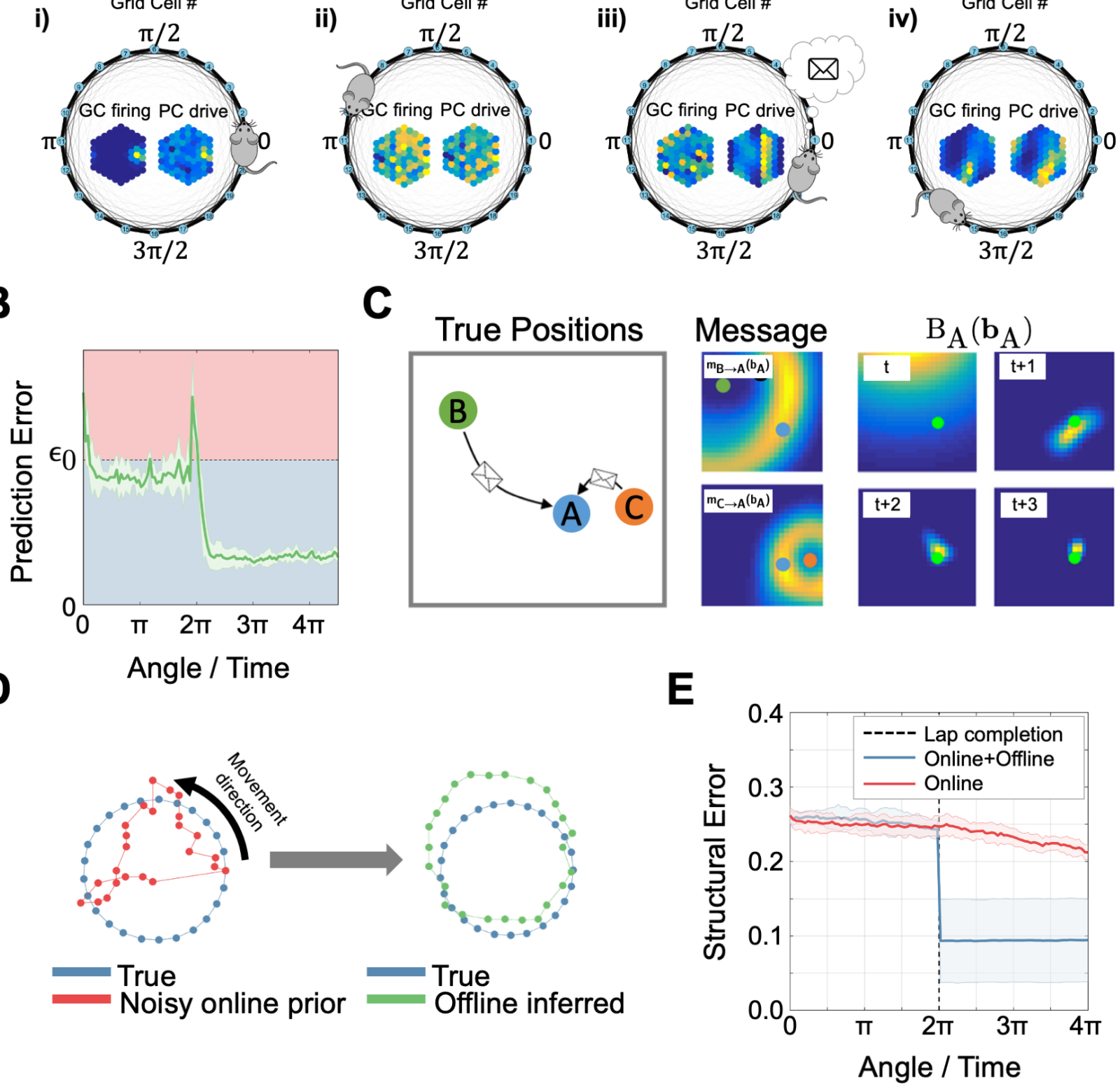
776

777

Figure 6. Principled message scheduling during offline inference generates sequences of place cell activity. A) Place cells (i.e. graph nodes representing conjunctions of environmental features) are connected via their pairwise potentials $\left(\psi_{A B}, \psi_{B C}\right)$, which penalize the mismatch between associative and metric pairwise distances $\left(\delta_{i j}\right.$ and $d_{i j}$ respectively). $(t=0)$ Environmental sensory input $\left(m_{S \rightarrow A}\left(b_{A}\right)\right.$ causes an update to the belief of place cell A (i.e. updating its synaptic weights to the grid sheet) by making it fire in a new location $b_{A}=1$. $(t=1)$ Place cell A sends a message to $\mathrm{B}$ expressing its belief over the location of $\mathrm{B}$, given its own (new) location and the associative distance $\delta_{A B}$, causing $\mathrm{B}$ to update its belief. ( $t=2)$ Messages from $B$ to $A$ and $C$ only cause $C$ to update its belief, so only $\mathrm{C}$ broadcasts at the next time-step. B) Examples of PC reactivation sequences in loopclosure task for different values of path integration noise $\sigma_{P I}^{2}$ (and therefore pairwise measurement confidence, since $w_{i j}=1 /\left(\sigma_{P C}^{2}+\sigma_{P I}^{2} \delta_{i j}\right)$; where $\left.\sigma_{P C}^{2}=1 e-4\right)$. Multiple local sequences can occur in interleaved fashion (Middle, grey shading) and become longer and smoother when pairwise measurements are less confident (Right; also E, F). C) The 'MaxEntropy' schedule (i.e., only the place cell with max entropy change fires in the next-step) converges faster than when all PCs broadcast messages at each time-step. D) Forward and reverse sequences occurred approximately equally often. 
A

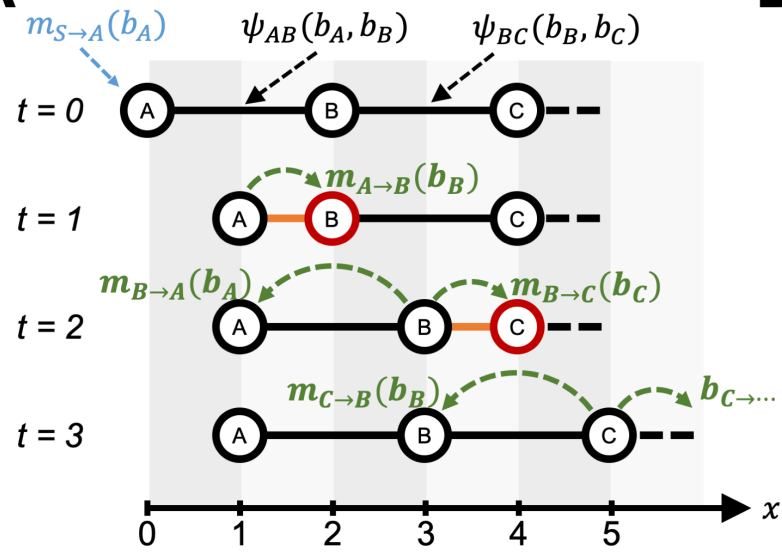

C

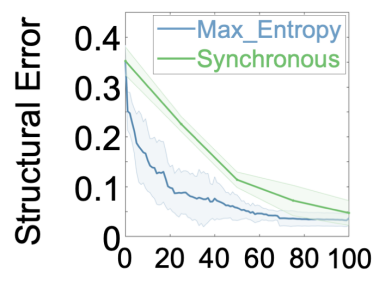

793
D

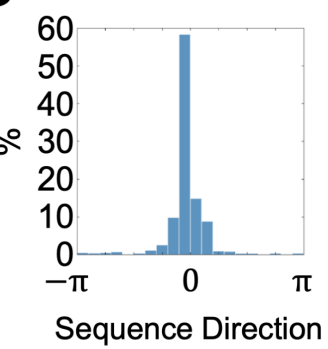

B
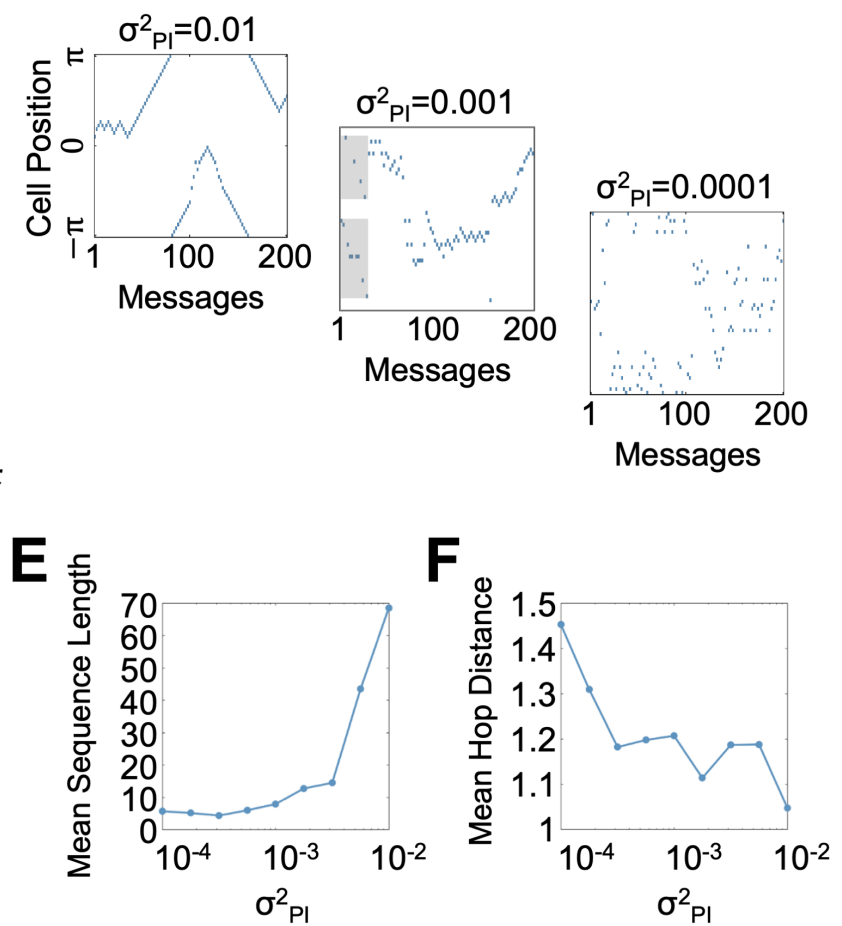
795 Figure 7. Schematic neural mechanism for probabilistic message passing (see also

796 Supplementary Video 2). A) Illustration in 1D. The broadcasting place cell $P_{B}$ sends a spike

797 to receiving place cell $\mathrm{P}_{\mathrm{R}}$ via recurrent connections in CA3, and also initiates a travelling

798 wave at the corresponding location on the grid cell module via their connections there. (Left)

799 No new learning occurs when the spike and travelling wave arrive at $P_{R}$ and its

800 corresponding grid location $\left(\mathrm{GC}_{7}\right)$ at the same time, as $\mathrm{P}_{\mathrm{R}}-\mathrm{GC}_{7}$ connection will already be

801 strong. (Middle) If the CA3 spike arrives at $\mathrm{P}_{\mathrm{R}}$ ahead of the travelling wave reaching $\mathrm{GC}_{7}$, the

802 synaptic associations of $\mathrm{P}_{\mathrm{R}}$ are adjusted towards the currently active $\mathrm{GC}_{5}$ (updating the

803 belief, by increasing $P_{R}-G C_{5}$ and decreasing $P_{R}-G C_{7}$, see $d W$, below). (Right) Same as

804 middle, except that $G C$ wave reaches $G_{7}$ before the $C A 3$ spike reaches $P_{R}$. B) Propagating

805 messages as travelling waves in mEC. A neural simulation of travelling waves with a

806 modified Laplacian diffusion kernel (Wave) closely approximates the probabilistic

807 propagation of activity (True), reflecting the accumulation of self-motion noise in the

808 broadening of the wave front. C) Comparison of the algorithmic and neural belief propagation performance. 


\section{A}
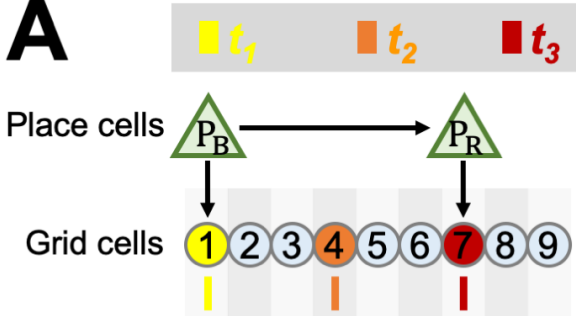

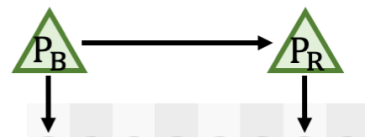

(1) 2 (4) 5078

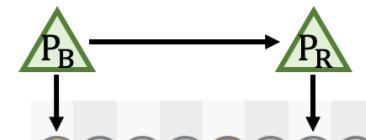

(1)(2) (3) 5 (6) 7 (8)

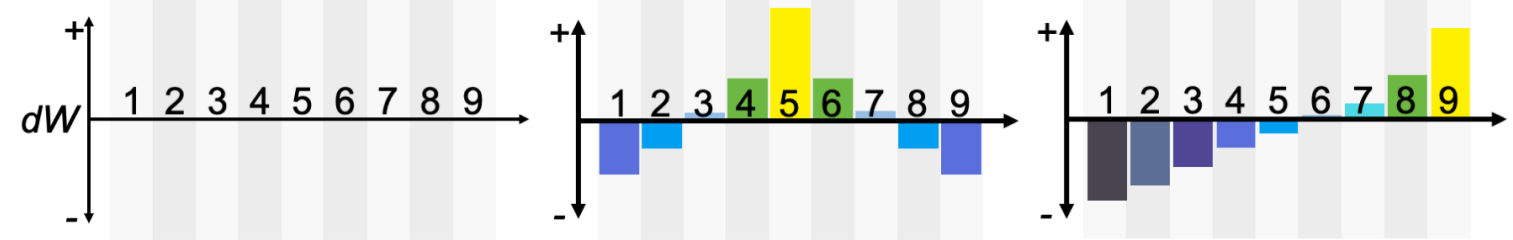

B

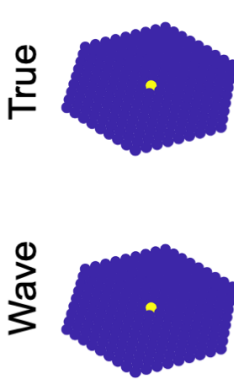

$\mathrm{T}_{0}$
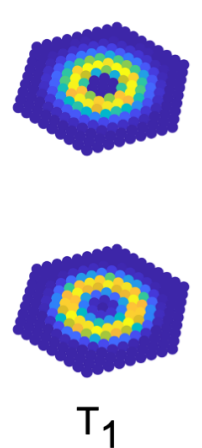
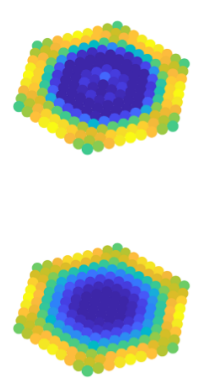

$\mathrm{T}_{2}$
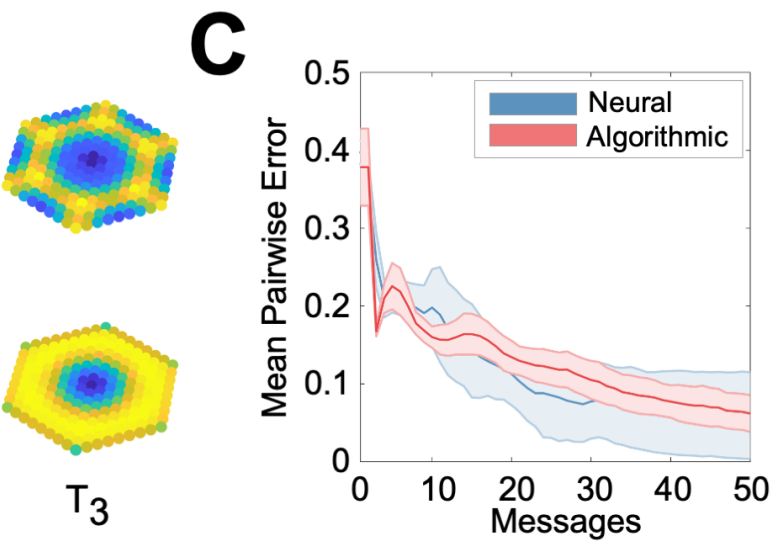
813 Figure S1 Implementational details of the online system. A The mapping from real to grid

814 cell space can be considered as the subtraction of a mapping vector $\left[i \cdot \boldsymbol{v}_{1}+j \cdot \boldsymbol{v}_{2}\right]$, where

815 each grid field can be described as a point on a 2D lattice with basis vectors $\boldsymbol{v}_{1}$ and $\boldsymbol{v}_{2}$. Each

816 grid field has associated with it a Voronoi domain, defined as the region within which any

817 point is closest to the corresponding grid field. When mapping from grid-to-real space, the

818 vector of the closest grid field is subtracted. B The wrapped Normal distribution is a

819 summation of the likelihoods of the current position estimate being at any one of an infinite

820 number of periodic tilings (here, five wrappings are shown). $\mathbf{C}$ The grid cells are connected

821 periodically to produce a 'twisted-torus' topology. D Illustration of the shifter cell mechanism.

822 Each 'readout' grid cell is connected to four 'shifter' grid cells and a single self-connecting

823 cell. E Illustration of the difference between wrapped and non-wrapped Gaussian

824 distributions. F Correspondence between the neural shifter cell mechanism and the

825 algorithmic transition function. G Pairwise distances between place fields can be inferred

826 from the strengths of Hebbian connections. H Mean entropy in the beliefs of each place cell

827 over their encoded location in grid space (encoded in place-grid cell connection weight

828 distributions decreases with iterations during offline inference.

A

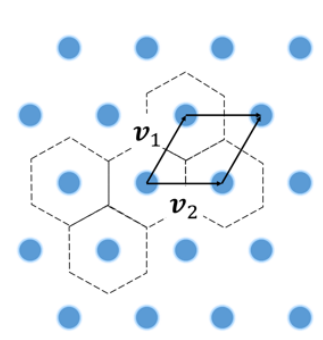

E

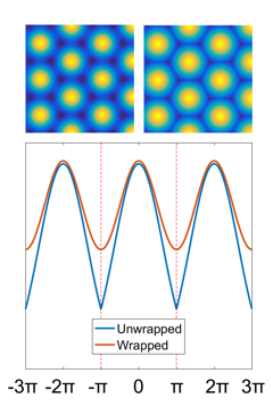

B

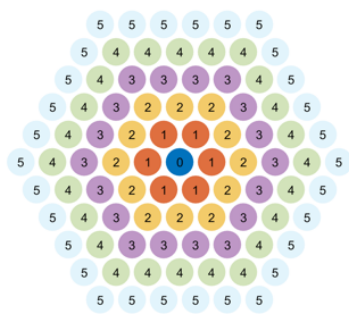

F

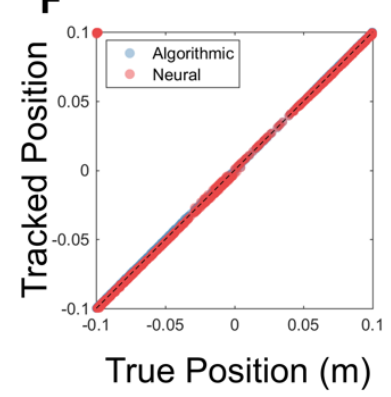

C

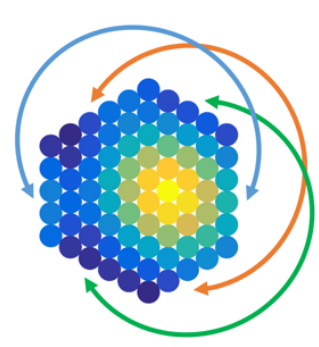

G

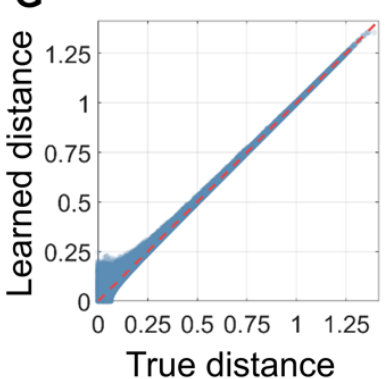

D

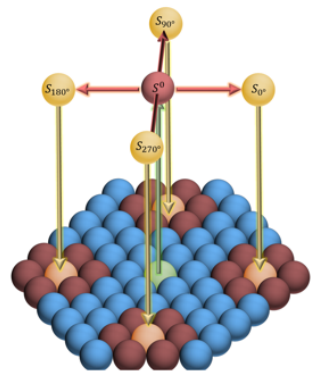

H

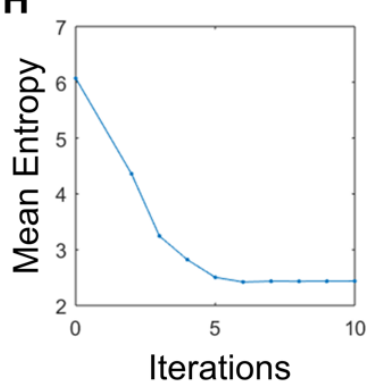


bioRxiv preprint doi: https://doi.org/10.1101/2020.08.07.241547; this version posted August 10, 2020. The copyright holder for this preprint (which was not certified by peer review) is the author/funder. All rights reserved. No reuse allowed without permission.

831

832 
833 Figure S2 A Convergence of grid patterns and place-grid cell weights over three periods

834 (columns). The place-grid cell weights (bottom row, colour denotes normalized connection

835 strength) converge fastest near to the boundaries (as seen in development; Muessig et al.,

$8362015)$ and corners of an environment, since trajectories through are more directionally

837 constrained. Top row shows corresponding grid patterns (colour denotes normalized firing

838 rate). B Convergence of stable grid patterns over sensory noise $\left(\sigma_{P C}\right)$ and path integration

839 noise $\left(\sigma_{P I}\right)$. Colour denotes grid score (Sargolini et al., 2006). $P$ indicates number of place

840 cells used in simulations. C-D Environmental deformations caused by prior structural beliefs.

841 C Place fields generated by the boundary vector cell model (Hartley et al., 2000). An

842 animal's perception of a trapezoidal environment (right) may be influenced by place-BVC

843 associations learned in a previous rectangular environment (left). In the trapezoid, simulated

844 place fields shift with the wall (D), causing similar distortion of the grid pattern.

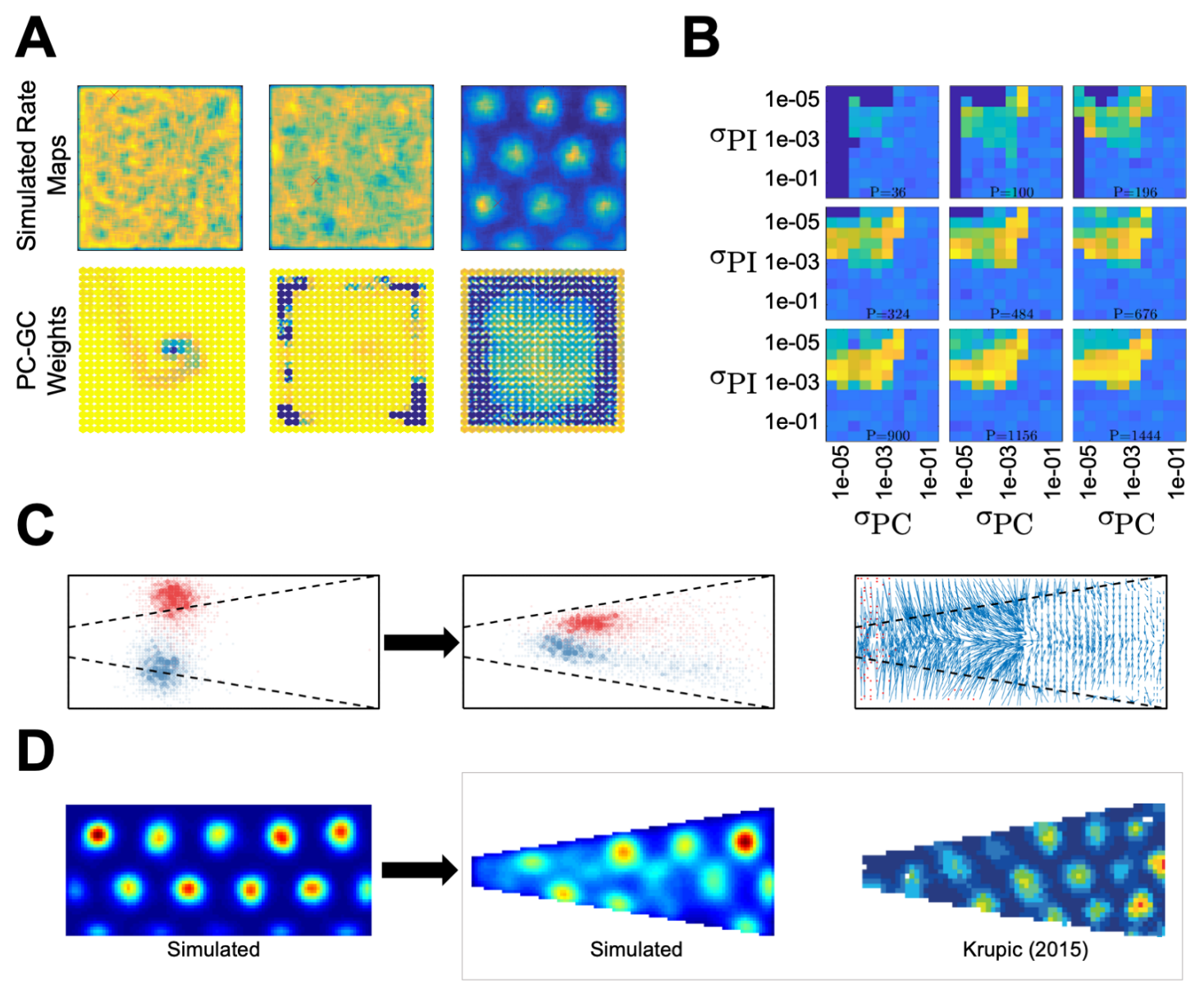


846 Figure S3. Grid distortions due to inhomogeneous environmental inputs in the online model.

847 A) When environmental inputs are concentrated in one corner of the environment (top-

848 middle panel), the resultant grid cell firing rate maps undergo a shearing distortion which

849 produces an orientation offset $\left(\mathrm{A}_{\min }\right.$; bottom-left panels). This offset increases with

850 experience, as grid cell firing becomes increasingly dependent on the maturing sensory

851 inputs (bottom-middle panel), matching experiments in which rats always entered the box at

852 the same corner (top-left panels; Stensola et al., 2015) The size of the experimental and

853 simulated offsets are similar (right pane/s). B) Simulated distortions based on an exponential

854 decay in place cell input from one corner as a function of the decay parameter (R). C)

855 Concentrated place field input along one wall and both corners (bottom left panel) causes

856 another distortion pattern (bottom right panel) also observed experimentally (top adapted

857 from Stensola et al., 2015).

858
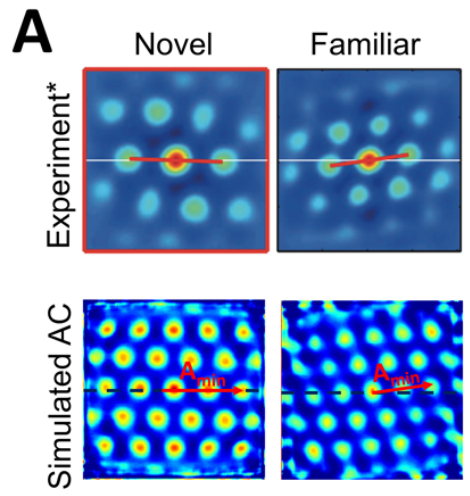

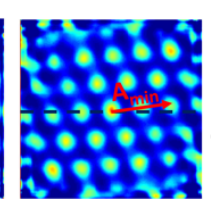

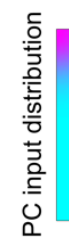

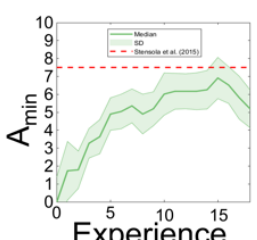

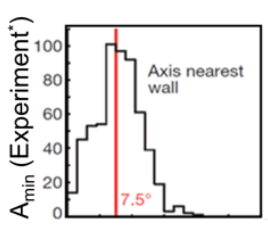

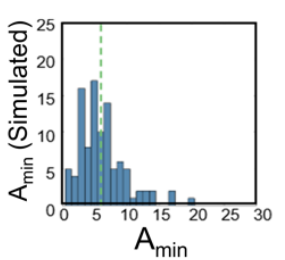

B
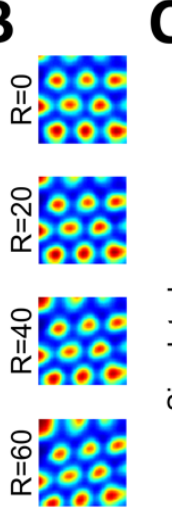

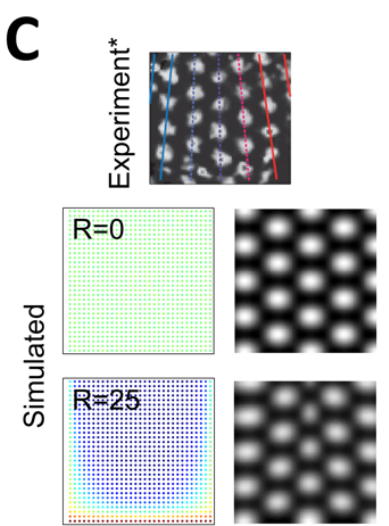


860 Figure S4 Mathematical analysis of shearing of the grid pattern due to inhomogeneous

861 environmental inputs. A The orientation resulting from the shearing operation can be

862 calculated by analysing the angles of the sheared hexagonal lattice describing the centroids

863 of the grid fields. B, C The analysis predicts that the orientation offset should be dependent

864 on both the strength of the place fields' density / firing rate imbalance $R$ (see Supplementary

865 Methods 1.7 and Fig. 2) (B) and their tuning widths (C).

A

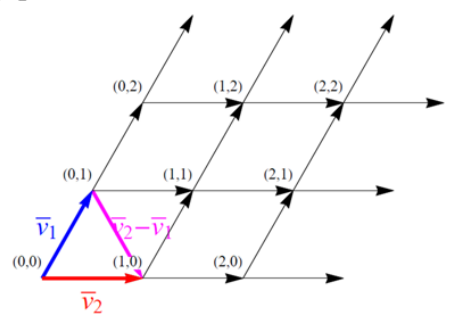

B

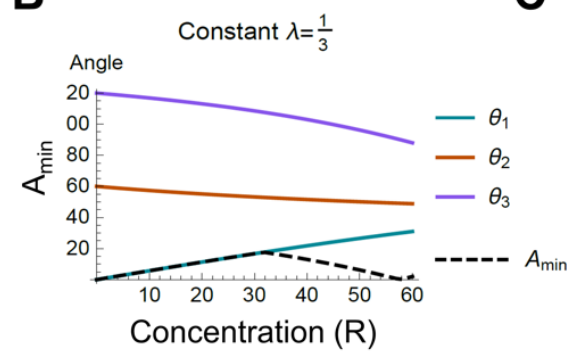

C

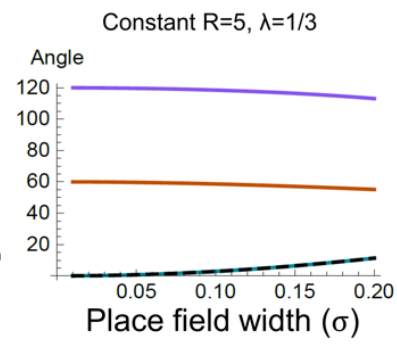


868 Figure S5 Anatomy of a SLAM system. The joint location-map probability distribution (A) is

869 represented in the firing rates and synaptic weights within the HPB-mEC system (B).

870

\section{A \\ $p\left(\boldsymbol{x},\left\{\boldsymbol{b}_{i}\right\}_{i=1}^{N G}\right)=$}

B

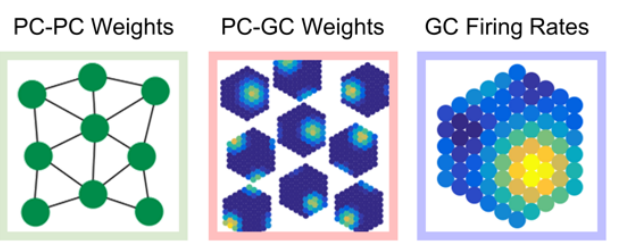


873 Figure S6 A Spring network analogy of the associative structure of an environment. Edge

874 'stiffness' is inversely proportional to the variance in the Gaussian observation. B 'Wrapping'

875 physical space to encoded location on the grid sheet. Each colour indicates the tiling of the

876 based grid sheet's domain in real space. C Pairwise distances near the edges of the

877 environment are overestimated due to under-sampling when the agent preferentially

878 explores the middle of an environment. Colours denote the distance of the pair of PCs $i$ and

$879 j$ from the walls of the $1 \times 1 \mathrm{~m}^{2}$ environment $d_{\text {wall }}=\sum_{p=i, j} \frac{1}{2}\left(\min \left(x_{p}, 1-x_{p}\right)+\min \left(y_{p}, 1-\right.\right.$

$\left.880 y_{p}\right)$ ). D Resulting local scale is proportional to the occupancy. C When the grid scale is smaller than the size of structure being encoded, we can think of 'wrapping' the structure onto the grid sheet. Here, colours denoted different tilings of the base metric tile (the Voronoi region of a given grid cell).

A

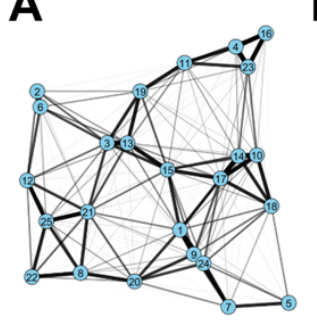

B

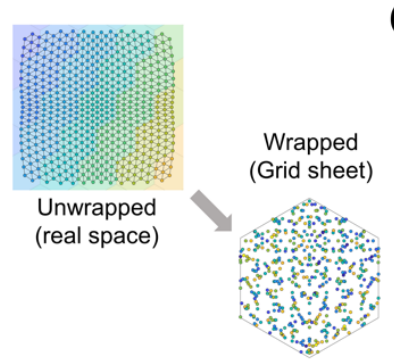

C

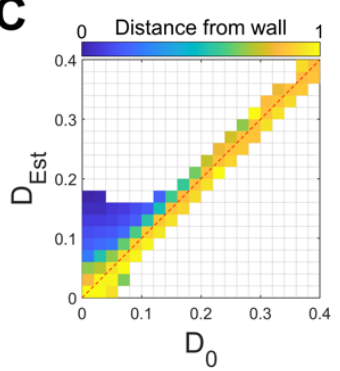

D

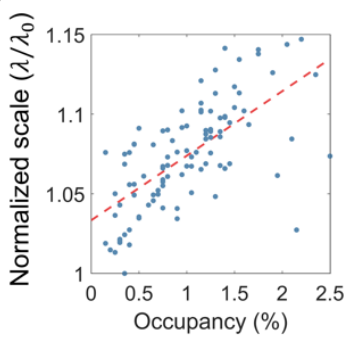

884

885

886 
Figure S7 An alternative view of the online and offline models. Place cells $P$ are driven by real-world stimulus $S$. During online exploration, the associative generative model is learned, but does not generate predictions. During offline inference, the metric generative model is corrected towards the predictions being generated by the online model, which becomes a surrogate for sensory stimuli as would be generated by the real world model.

\section{Online}

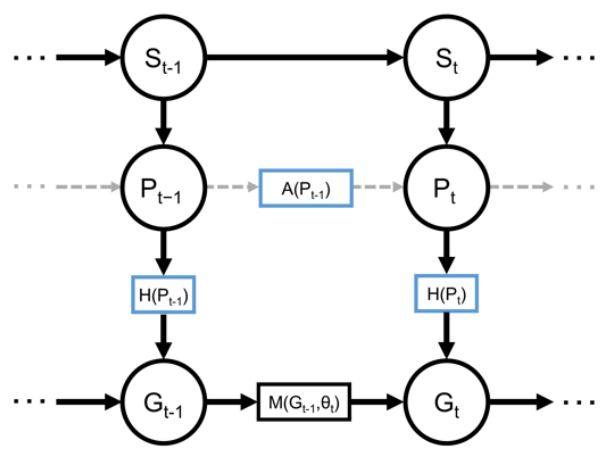

\section{Offline}

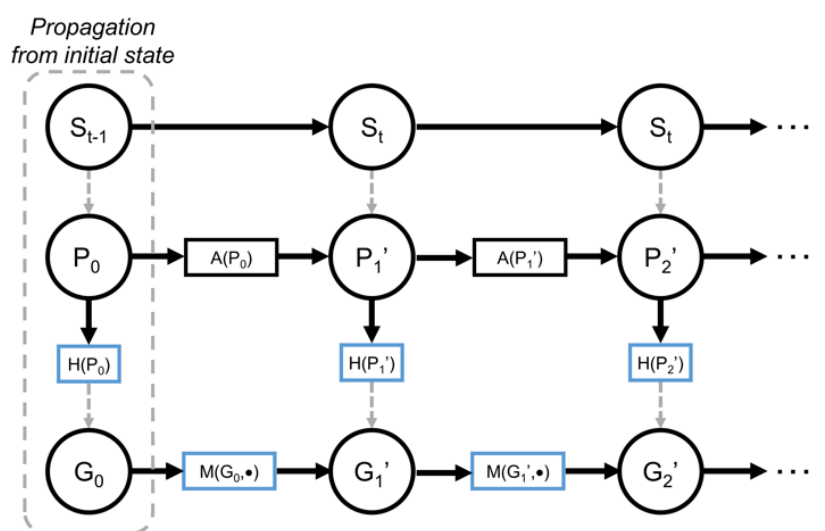


bioRxiv preprint doi: https://doi.org/10.1101/2020.08.07.241547; this version posted August 10, 2020. The copyright holder for this preprint (which was not certified by peer review) is the author/funder. All rights reserved. No reuse allowed without permission. 
910 localization and learning in the HPC/mEC.

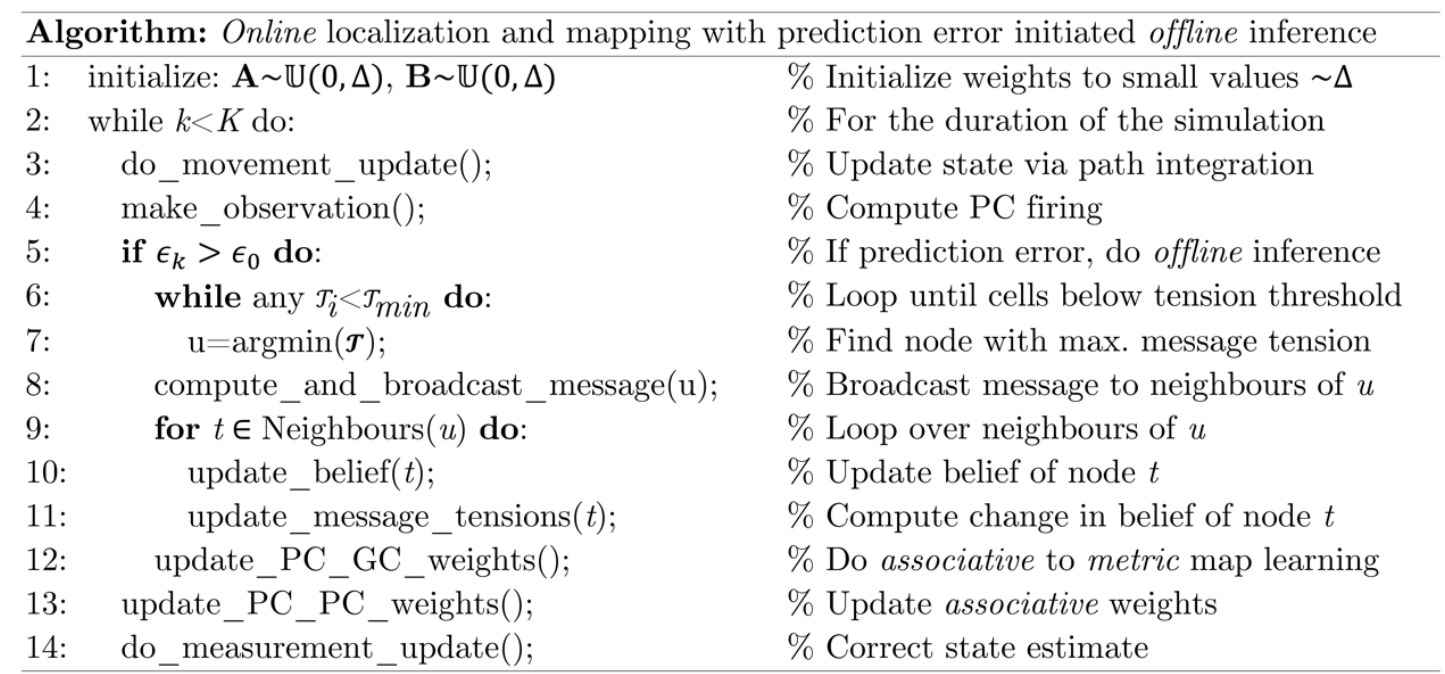

